\title{
Mode interaction in perfect and imperfect thin-walled I-section struts susceptible to global buckling about the strong axis
}

\author{
Elizabeth L. Liu, M. Ahmer Wadee* \\ Department of Civil and Environmental Engineering, Imperial College London, \\ South Kensington Campus, London SW7 2AZ, UK
}

\begin{abstract}
A recently developed analytical model for a perfect I-section strut experiencing a nonlinear interaction between local buckling and global buckling about the strong axis is enhanced and subsequently extended. The initial enhancement is achieved by devising a simplified and calibrated model that provides an improved prediction of the local buckling load. A purely numerical model is then constructed within the commercial finite element (FE) software ABAQUS for validation purposes and excellent comparisons are observed, demonstrating that the analytical model is considerably improved on previous work. The model for interactive buckling is then developed subsequently to include the effects of global and local geometric imperfections, which are introduced individually and in combination. The strut is found to be sensitive to all considered shapes of imperfection and the combined imperfection case correlates excellently with an equivalent FE model, particularly in the neighbourhood of the secondary instability that leads to mode interaction. This demonstrates that the enhanced analytical model predicts the actual load carrying capacity and the structural mechanics accurately.
\end{abstract}

Keywords: Analytical modelling; Mode interaction; Imperfection sensitivity; Nonlinear mechanics; Strong axis global buckling.

\section{Introduction}

It is well known that struts and columns constructed from thin-walled elements under compression are practically vulnerable to a range of different elastic buckling instabilities [1, $2,3,4]$. Particularly in cases where global and local instability modes have similar critical loads, nonlinear modal interactions are often observed in the post-buckling response of such structures. This can lead to unstable behaviour and may cause a significant decrease in load carrying capacity when compared to the predicted capacities for each individual mode [5, 6 , $7,8,9]$. Several studies on similar components exist that employ experimental techniques

\footnotetext{
*Corresponding author

Email addresses: elizabeth.1iu07@imperial.ac.uk (Elizabeth L. Liu), a.wadee@imperial.ac.uk (M. Ahmer Wadee)
} 
and finite element (FE) analysis to study global and local mode interaction in thin-walled carbon steel and stainless steel columns $[10,11,12,13]$. It is also the case that such systems have previously exhibited a high sensitivity to initial global and local geometric imperfections [14, 15, 16, 17, 18]. However, thin-walled structures have attractively high strength to weight ratios and are therefore used extensively in industry, particularly in the maritime, aeronautical and civil engineering sectors [1, 19]. It is essential therefore that continually improved understanding of the physical behaviour of these practically important component types is developed.

In the authors' previous work, an I-section strut with a rigid flange-web connection was investigated using an analytical approach where nonlinear mode interaction was observed between the critical global buckling mode about the strong axis and a local plate buckling mode [20]. The post-buckling behaviour of the strut was found to be highly unstable and led to lower load carrying capacities than would be anticipated for either individual buckling mode. A changing wavelength was also observed in the local out-of-plane displacement profile of the strut flange and web elements with results being compared to an equivalent model constructed in the commercial FE package ABAQUS [21], giving reasonable comparisons particularly at and immediately beyond the secondary bifurcation point where interactive buckling is triggered.

The global buckling load was well predicted by the analytical model, matching the FE prediction to within 1\%. However, the local buckling load was less well predicted, largely owing to the relative uncertainty of the actual rotational stiffness provided by the flange-web connection, previously assumed to be completely rigid in the analytical model. The initial aim of the current work is to enhance the previous model by providing a more accurate analytical prediction of the local buckling load. An analytical model is duly presented that describes the behaviour of an I-section strut experiencing local buckling only. A parameter $\kappa$ determines the actual level of rigidity provided by the flange-web connection. This parameter is calibrated by comparing the analytically predicted local buckling load to an equivalent FE model formulated in the commercial package ABAQUS [21]. This calibrated value is then used to enhance the previous model of a perfect, long strut, where global buckling is critical [20], which interacts with the local buckling mode resulting in significantly improved comparisons with the FE model for the interactive buckling case.

Moreover, in previous work, only global geometric imperfections were introduced into the analytical formulation in the form of initial stress-relieved out-of-straightness deformations. The study that was conducted for the fully rigid flange-web joint is revisited presently with the calibration implemented. The strut is still found to be sensitive to initial out-of-straightness geometric imperfections, which results in a decreasing ultimate load as the magnitude of the initial imperfection is increased. The currently enhanced analytical model is then extended further to include initial local imperfections, individually and in combination with the global imperfection. An imperfection sensitivity study is then conducted for a variety of out-of-plane imperfection profiles. The model shows sensitivity to all shapes of the local imperfections prescribed, with a greater decrease in the ultimate load for localized imperfections reflecting previous work on other similarly behaving structural systems $[22,23,24]$. This finding is also validated by the FE model showing excellent 
comparisons, particularly in the initial post-buckling range. The study has the potential to be extended to determine the model parameter ranges where interactive buckling is significant; this is currently under development and the authors hope to report on this in due course.

\section{Analytical formulation}

The strut studied currently is shown in Fig. 1 and is assumed to be restrained from
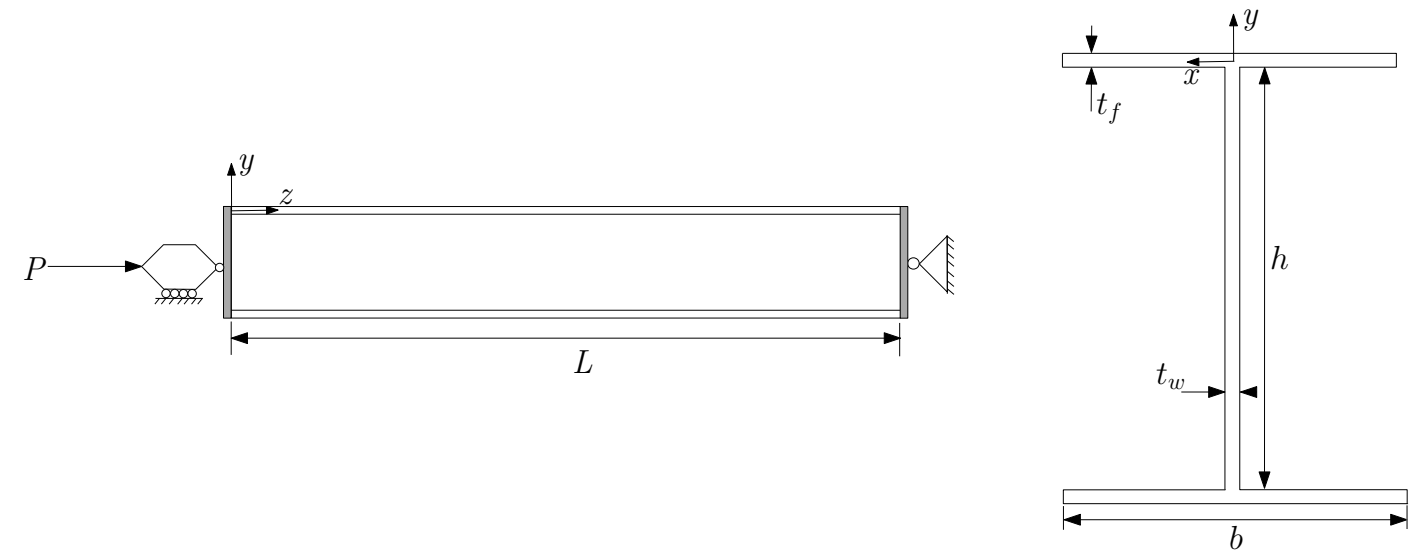

Figure 1: An I-section under axial loading $P$, shown using the elevation (left) and the cross-section (right). The ends are simply supported and a rigid end plate transfers the load equally to the flanges from the supports.

buckling globally about the weaker $y$-axis. The formulation of the analytical model begins by defining the displacement functions for global buckling modes, as well as the local buckling modes in the in-plane $u$ and out-of-plane $w$ directions. Note that deflections in the third dimension $v$ are very small and are therefore neglected currently [2].

\subsection{Global buckling modes}

The two global degrees of freedom defined in previous work [20], so-called 'sway' and 'tilt', are shown in Fig. 2. and are defined thus:

$$
W(z)=q_{s} L \sin \left(\frac{\pi z}{L}\right), \quad \theta=q_{t} \pi \cos \left(\frac{\pi z}{L}\right) .
$$

The difference between the two modal amplitudes $q_{s}$ and $q_{t}$ allows shear strains to be accounted for in the strut, which is essential to capture any interactive behaviour occurring during post-buckling [25]. In order to include an initial, stress-relieved, out-of-straightness as a global imperfection, the functions $W_{0}$ and $\theta_{0}$ are also introduced:

$$
W_{0}(z)=q_{s 0} L \sin \left(\frac{\pi z}{L}\right), \quad \theta_{0}=q_{t 0} \pi \cos \left(\frac{\pi z}{L}\right),
$$

where $q_{s 0}$ and $q_{t 0}$ are the respective amplitudes, also shown in Fig. 2, corresponding to those from the sway and tilt degrees of freedom. 

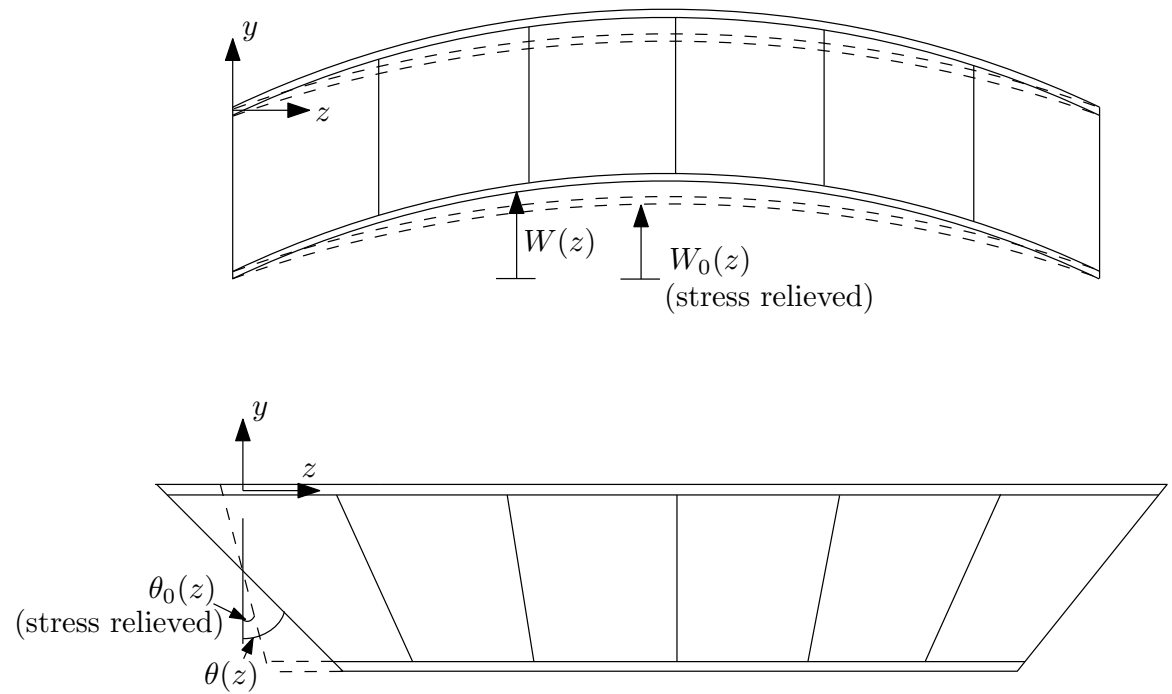

Figure 2: Degrees of freedom for global buckling, so-called 'sway' $W$ and 'tilt' $\theta$ modes along with the respective initial global imperfection lateral displacement $W_{0}$ and plane section rotation $\theta_{0}$.

\subsection{Local buckling modes}

For the local buckling displacements, there are up to six separate functions that need to be introduced, depending on whether global or local buckling is critical. Figure 3 shows these displacement functions and are defined thus:

$$
\begin{aligned}
& u_{f l i}(x, z)=u_{f i}(z), \quad u_{w l}(y, z)=-\left(\frac{y}{h}\right) u_{w 1}(z)+\left(1+\frac{y}{h}\right) u_{w 2}(z), \\
& w_{f l i}(x, z)=f_{i}(x) w_{f i}(z), \quad w_{w l}(y, z)=g_{1}(y) w_{w 1}(z)+g_{2}(y) w_{w 2}(z),
\end{aligned}
$$

for the in-plane flange and web displacements, as well as the out-of-plane flange and web displacements respectively. Here, $i=\{1,2\}$ to account for the respective deflections of both the bottom and top flanges, as shown in Fig. 3. For the case where global buckling is critical, it is assumed that the less compressed top flange remains undeflected throughout post-buckling, as shown in Fig. 4. Hence, in this case, all local displacement profiles associated with the less compressed flange (i.e. with subscript 2) are assumed to be zero, reducing the number of local buckling displacement functions from eight to four.

The functions $f_{i}(x)$ and $g_{i}(y)$ are selected such that they satisfy the boundary conditions for each individual component, as well as giving a good representation of the deflected shape of the component. The out-of-plane deflection profile of the flange is approximated to be linear, resulting in the function $f_{i}(x)=(-1)^{i}(2 x / b)$ [20]. The out-of-plane displacement of the web is defined such that when local buckling only is present, i.e. when $w_{w 1}(z)=$ $w_{w 2}(z)$, the summation $\left[g_{1}(y)+g_{2}(y)\right]$ gives a symmetric representation of the out-of-plane displacement profile of the web, as shown in Fig. 3. However, when global buckling is critical and $w_{w 2}(z)=0$, the out-of-plane displacement begins to resemble the representation in 

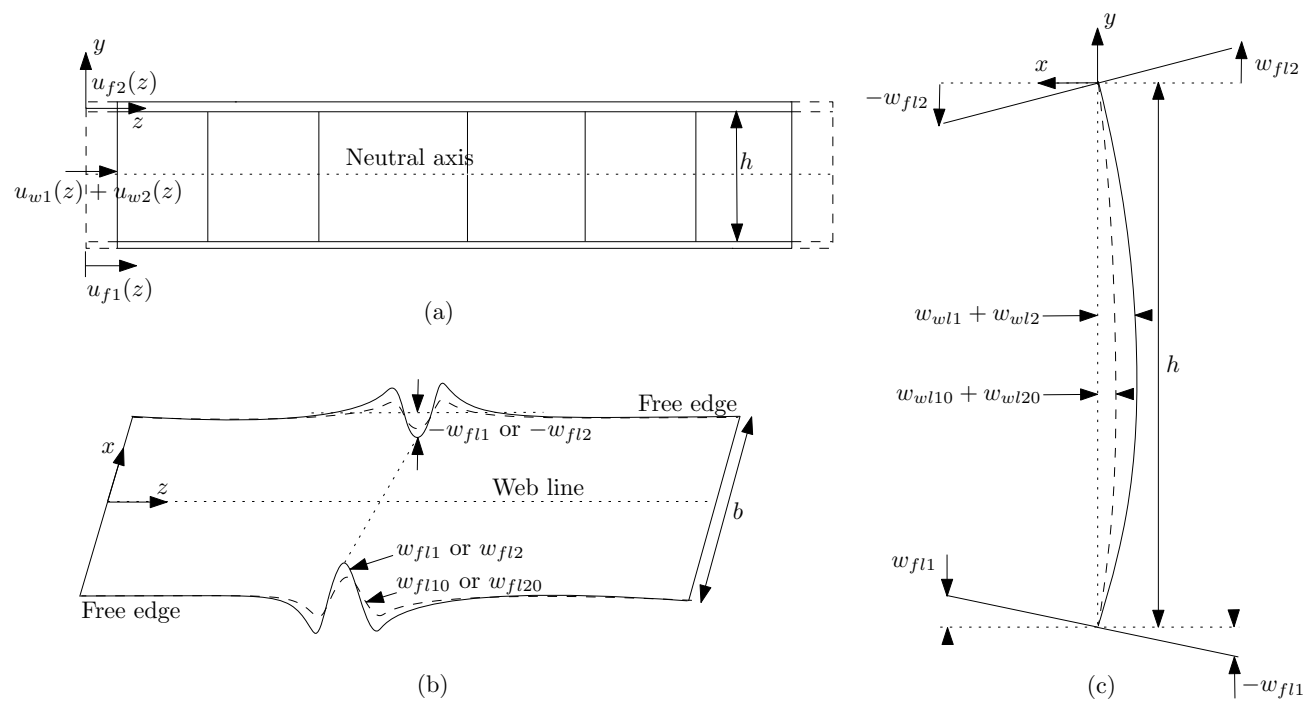

Figure 3: Local buckling modal components representing in-plane and out-of-plane displacements for the flange and web, alongside those of out-of-plane local imperfections.

Fig. 4. Thus, the function $w_{w l}$ can be written as:

$$
w_{w l}(z)=g_{1}(y) w_{w 1}(z)+g_{2}(y) w_{w 2}(z)
$$

where

$$
g_{i}(y)=(-1)^{i}\left[\frac{\pi}{2} \cos \left(\frac{\pi y}{h}\right)-\left(\frac{\pi}{2}+\frac{\pi y}{h}\right)\right]-\sin \left(\frac{\pi y}{h}\right)
$$

the functions $g_{i}(y)$ being defined such that they are solutions of the Euler strut equation with unequal end rotations [26]. Note that for the case where local buckling only is present and $w_{w}(z)=w_{w 1}(z)=w_{w 2}(z)$, the out-of-plane displacement for the web becomes symmetric about the mid-depth of the web (where $y=-h / 2$ ), thus:

$$
w_{w l}(y, z)=2 \sin \left(\frac{\pi y}{h}\right) w_{w}(z) .
$$

The local out-of-plane imperfection shapes are then introduced for the flange and the web as the following functions respectively:

$$
\begin{aligned}
w_{f l i 0}(x, z) & =f_{i}(x) w_{f 0}(z)=f_{i}(x)\left\{A_{0} \operatorname{sech}[\alpha(z-\psi) / L] \cos [\beta \pi(z-\psi) / L]\right\}, \\
w_{w l 0}(y, z) & =g_{1}(y) w_{w 10}(z)+g_{2}(y) w_{w 20}(z) \\
& =\left[A_{1} g_{1}(y)+A_{2} g_{2}(y)\right] \operatorname{sech}[\alpha(z-\psi) / L] \cos [\beta \pi(z-\psi) / L]
\end{aligned}
$$

which are derived from a leading order approximation of the deflected shape of a strut on a softening elastic foundation in the post-buckling range [27]. In this function, $z=[0, L]$, the parameter $A_{0}$ determines the amplitude of the imperfection at midspan for the flange with $A_{1}$ and $A_{2}$ determining the same for the web. The quantity $\beta$ determines the number 


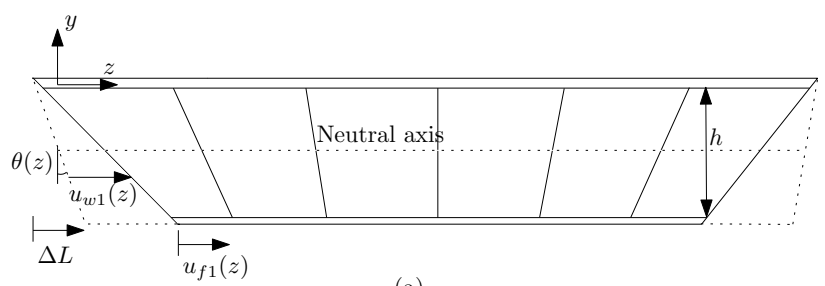

(a)

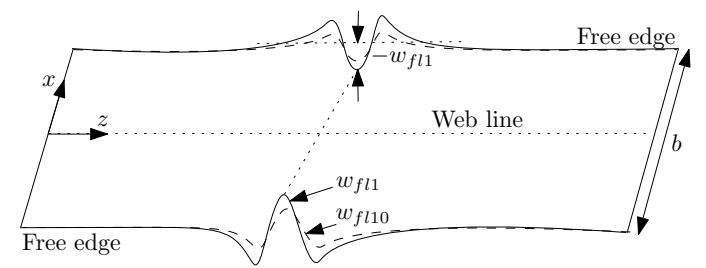

(b)

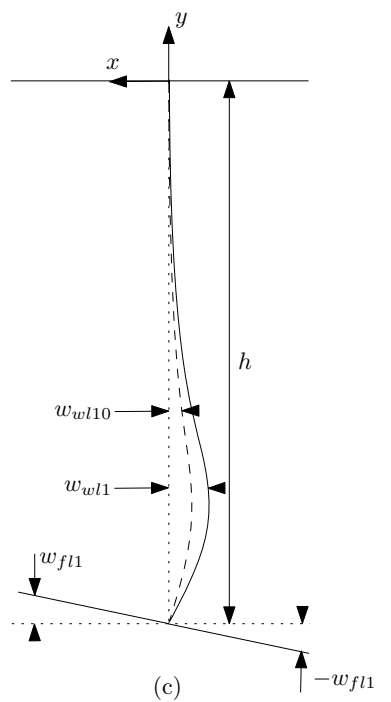

Figure 4: In-plane and out-of-plane displacements for the flange and web describing the local buckling modes, along with out-of-plane local imperfections, for the case where global buckling is critical.

of half waves that appear along the length of the imperfection, which must always be an odd integer such that the expression satisfies reflective symmetry that is demonstrated to be the worst case if global and local buckling interact [22]; the parameter $\psi$ is set to be $L / 2$, to maintain the symmetry condition at the strut midspan for the same reason. The parameter $\alpha$ determines the amount of localization, such that if $\alpha=0$, the expression would give a purely periodic imperfection shape. The selected local imperfection function has also been shown in the literature to provide a good representation of the worst case imperfection shape for global-local mode interaction problems [22]. The shape of the local out-of-plane imperfection is shown in Fig. 5 with varying $\alpha$ and $\beta$ values and a normalized amplitude of unity.

\subsection{Total potential energy}

The total potential energy $V$ is the summation of the total strain energy $U$, minus the work done by the external load. Each component is presented currently in the most general form and simplified at a later stage for each specific application. Firstly, the strain energy stored in bending $U_{B}$ due to global buckling about the strong axis is given by the expression:

$$
U_{B}=2 \int_{0}^{L} \frac{E I_{f}}{2}\left[\frac{\mathrm{d}^{2}}{\mathrm{~d} z^{2}}\left(W-W_{0}\right)\right]^{2} \mathrm{~d} z,
$$

where $I_{f}=b t_{f}^{3} / 12$ and is the second moment of area of the flange about its local $x$-axis. Note that the global bending energy has been multiplied by two to account for both top and bottom flanges. The local bending energy contributions from the flange $U_{b f}$ and from 


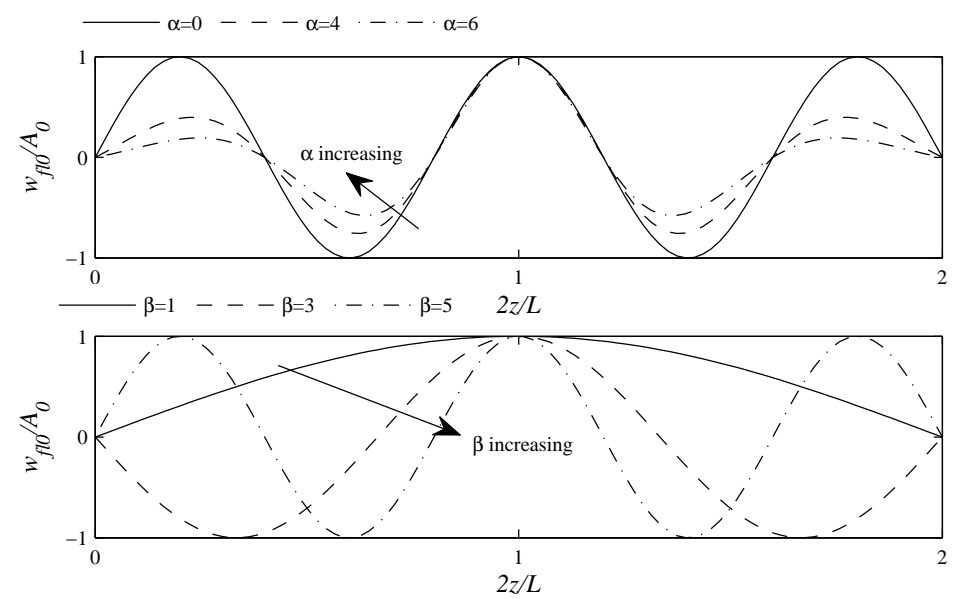

Figure 5: Local imperfection shapes $w_{f l 0}$ with (top) a varying localization parameter $\alpha$ for a fixed $\beta$ value and (bottom) a varying wave number parameter $\beta$ when $\alpha=0$.

the web $U_{b w}$ are determined with the respective expressions:

$$
\begin{aligned}
U_{b f i}=\frac{D_{f}}{2} \int_{0}^{L} \int_{-b / 2}^{b / 2}\left\{\left(\frac{\partial^{2}\left(w_{f l i}-w_{f l i 0}\right)}{\partial x^{2}}+\frac{\partial^{2}\left(w_{f l i}-w_{f l i 0}\right)}{\partial z^{2}}\right)^{2}\right. \\
\left.\quad-2(1-\nu)\left[\frac{\partial^{2}\left(w_{f l i}-w_{f l i 0}\right)}{\partial x^{2}} \frac{\partial^{2}\left(w_{f l i}-w_{f l i 0}\right)}{\partial z^{2}}-\left(\frac{\partial^{2}\left(w_{f l i}-w_{f l i 0}\right)}{\partial x \partial z}\right)^{2}\right]\right\} \mathrm{d} x \mathrm{~d} z, \\
U_{b w}=\frac{D_{w}}{2} \int_{0}^{L} \int_{-h}^{0}\left\{\left(\frac{\partial^{2}\left(w_{w l}-w_{w l 0}\right)}{\partial y^{2}}+\frac{\partial^{2}\left(w_{w l}-w_{w l 0}\right)}{\partial z^{2}}\right)^{2}\right. \\
\left.\quad-2(1-\nu)\left[\frac{\partial^{2}\left(w_{w l}-w_{w l 0}\right)}{\partial y^{2}} \frac{\partial^{2}\left(w_{w l}-w_{w l 0}\right)}{\partial z^{2}}-\left(\frac{\partial^{2}\left(w_{w l}-w_{w l 0}\right)}{\partial y \partial z}\right)^{2}\right]\right\} \mathrm{d} y \mathrm{~d} z,
\end{aligned}
$$

where $i=\{1,2\}$ to account for the effect of both the bottom flange and the top flange in turn. The quantities $D_{f}=E t_{f}^{3} /\left[12\left(1-\nu^{2}\right)\right]$ and $D_{w}=E t_{w}^{3} /\left[12\left(1-\nu^{2}\right)\right]$ represent the flexural rigidities of the flange and web respectively.

The membrane energy from direct strain in the flanges can be found from the expression:

$$
U_{m \epsilon f}=\frac{1}{2} E t_{f} \int_{0}^{L} \int_{-b / 2}^{b / 2}\left(\epsilon_{z 1}^{2}+\epsilon_{z 2}^{2}\right) \mathrm{d} x \mathrm{~d} z
$$

where the general expression for the direct strain in the flanges is thus:

$$
\epsilon_{z i}=(-1)^{(i+1)} \frac{h}{2} \frac{\mathrm{d}}{\mathrm{d} z}\left(\theta-\theta_{0}\right)-\Delta+\left\{\frac{\partial u_{f l i}}{\partial z}+\frac{1}{2}\left[\left(\frac{\partial w_{f l i}}{\partial z}\right)^{2}-\left(\frac{\partial w_{f l i 0}}{\partial z}\right)^{2}\right]\right\} .
$$


The variable $\theta$ is the rotation relating to the tilt degree of freedom as defined earlier and $\Delta$ is the strain due to end-shortening from pure compression in the strut. The three additional terms are only present when the considered flange is in compression, and are obtained from von Kármán plate theory [28].

The direct strain contribution to the strain energy stored in the web is defined by the expression:

$$
U_{m \epsilon w}=\frac{1}{2} E t_{w} \int_{0}^{L} \int_{-h}^{0} \epsilon_{z w}^{2} \mathrm{~d} y \mathrm{~d} z
$$

where the direct strain in the web is given by the following expression:

$$
\epsilon_{z w}=\frac{\partial u_{t}}{\partial z}-\Delta+\frac{\partial u_{w l}}{\partial z}+\frac{1}{2}\left(\frac{\partial w_{w l}}{\partial z}\right)^{2}-\frac{1}{2}\left(\frac{\partial w_{w l 0}}{\partial z}\right)^{2}
$$

with $u_{t}=-(y+h / 2)\left(\theta-\theta_{0}\right)$. The shear strain contribution to the strain energy stored in the flanges is defined:

$$
U_{m \gamma f}=\frac{1}{2} G t_{f} \int_{0}^{L} \int_{-b / 2}^{b / 2}\left(\gamma_{x z 1}^{2}+\gamma_{x z 2}^{2}\right) \mathrm{d} x \mathrm{~d} z,
$$

where the general flange shear strain term $\gamma_{x z i}$ is:

$$
\gamma_{x z i}=\frac{\partial u_{f l i}}{\partial x}+\frac{\partial w_{f l i}}{\partial z} \frac{\partial w_{f l i}}{\partial x}-\frac{\partial w_{f l i 0}}{\partial z} \frac{\partial w_{f l i 0}}{\partial x} .
$$

This expression can be simplified by noting that the flange in-plane displacement $u_{f l i}$ is uniform in $x$ and hence the first term is zero. The contribution from the shear strain to the web strain energy is given by the expression:

$$
U_{m \gamma w}=\frac{1}{2} G t_{w} \int_{0}^{L} \int_{-h}^{0} \gamma_{y z}^{2} \mathrm{~d} y \mathrm{~d} z
$$

where the shear strain in the web $\gamma_{y z}$ is defined:

$$
\gamma_{y z}=\frac{\partial}{\partial z}\left(W-W_{0}\right)-\left(\theta-\theta_{0}\right)+\frac{\partial u_{w l}}{\partial y}+\frac{\partial w_{w l}}{\partial y} \frac{\partial w_{w l}}{\partial z}-\frac{\partial w_{w l 0}}{\partial y} \frac{\partial w_{w l 0}}{\partial z} .
$$

Note that in Equation (16) the first two terms that appear in Equation (18) are omitted from the shear strain expression for the flange since the difference between the $q_{s}$ and $q_{t}$ terms is very small in the flange because it is thin.

The work done by the load term contributing to the total potential energy is expressed as the load $P$ multiplied by the distance moved by the load in the same direction and is written as:

$$
P \mathcal{E}=P \int_{0}^{L}\left[\frac{q_{s}^{2} \pi^{2}}{2} \cos ^{2}\left(\frac{\pi z}{L}\right)-\frac{1}{2}\left(\dot{u}_{w 1}+\dot{u}_{w 2}\right)+\Delta\right] \mathrm{d} z,
$$


where the respective contributions are displacements from the global mode, the local mode and from pure axial compression. The total potential energy $V$ is given by the sum of all the strain energy terms presented, minus the work done by the load as follows:

$$
V=U_{B}+U_{b f 1}+U_{b f 2}+U_{b w}+U_{m \epsilon f 1}+U_{m \epsilon f 2}+U_{m \epsilon w}+U_{m \gamma f 1}+U_{m \gamma f 2}+U_{m \gamma w}-P \mathcal{E} .
$$

Linear eigenvalue analysis is used to determine the analytical expression for the global strong axis buckling load of the perfect strut. The Hessian matrix $\mathbf{V}_{i j}^{\mathrm{F}}$, evaluated on the fundamental (pre-buckling) path $\mathrm{F}$ is given by:

$$
\mathbf{V}_{i j}^{\mathrm{F}}=\left[\begin{array}{cc}
\left.\frac{\partial^{2} V}{\partial q_{s}^{2}}\right|^{\mathrm{F}} & \left.\frac{\partial^{2} V}{\partial q_{s} \partial q_{t}}\right|^{\mathrm{F}} \\
\left.\frac{\partial^{2} V}{\partial q_{t} \partial q_{s}}\right|^{\mathrm{F}} & \left.\frac{\partial^{2} V}{\partial q_{t}^{2}}\right|^{\mathrm{F}}
\end{array}\right],
$$

where all the local displacements associated with $w_{f l i}, w_{w l}, u_{f l i}$ and $u_{w l}$ are zero. The matrix is singular at the global critical load $P_{o}^{\mathrm{C}}$, the expression for which can be written thus:

$$
P_{o}^{\mathrm{C}}=\frac{2 E I_{f} \pi^{2}}{L^{2}}+\frac{G\left(h t_{w}+6 b t_{f}\right)}{1+6 b t_{f} /\left(h t_{w}\right)+12 G L^{2} /\left(E \pi^{2} h^{2}\right)} .
$$

\subsection{Modelling the flange-web joint}

A relationship between the out-of-plane displacements of the web and the flange can be established by considering the ratio between the two deflections, using a scaling parameter $\kappa$ thus:

$$
w_{w i}(z)=\kappa w_{f i}(z) .
$$

The parameter $\kappa$ is determined by the rigidity of the joint connecting the web to the flanges. For example, if the joint is assumed to be fully rigid, and free to rotate as a rigid body, it can be deduced that $\kappa=\kappa_{0}=-h /(\pi b)$ by equating the slope of their respective displacement functions, evaluated at this location. This assumption is used in the authors' previous work [20]. However, if the joint is not fully rigid, $|\kappa|<\left|\kappa_{0}\right|$, since the web would deflect less in sympathy with the more slender flange during post-buckling. The advantages of incorporating the parameter $\kappa$ are two-fold: firstly, the number of functions to be solved are reduced further by defining the functions $w_{1}=w_{f 1}=w_{w 1} / \kappa, w_{2}=w_{f 2}=w_{w 2} / \kappa$; secondly, the parameter can be adjusted to model different rigidities of the flange-web connection. In all the following derivations, the parameter $\kappa$ is implemented. Note that, in a similar way, $\kappa$ can also be applied to the local imperfection profiles, such that:

$$
w_{w i 0}(z)=\kappa w_{f i 0}(z),
$$

which, in turn, reduces the number of imperfection functions that need to be introduced. 


\section{Pure local buckling}

Using the general form of each energy component detailed above, the total potential energy can be formulated to describe the response of the perfect strut, for a situation where only local buckling is present. To consider the perfect case strut, all initial geometric imperfections are assumed to be zero in the following formulation. The appropriate in-plane and out-of-plane displacement functions defined earlier for the case where local buckling is critical are substituted into $V$. When only local buckling is present, it may be assumed that the deformation profile of the strut is symmetric about the strong axis, i.e. $w=w_{f 1}=w_{f 2}$, $u=u_{f 1}=u_{f 2}$ and $\kappa w=w_{w 1}=w_{w 2}$. Additionally, generalized coordinates describing global buckling, $q_{s}$ and $q_{t}$, are assumed to be zero. Note that the total potential energy is presented in a non-dimensional form, with respect to the strut half-length, i.e. $\tilde{z}=2 z / L$, out-of-plane displacements have also been rescaled as $\tilde{w}=2 w / L$ and $\tilde{u}=2 u / L$ accordingly. The total potential energy for the perfect case strut where only local buckling is present can be written thus:

$$
\begin{aligned}
V=\int_{0}^{2} & \frac{1}{32 L}\left\{32\left[D_{f}\left(\left\{f_{1}^{2}+f_{2}^{2}\right\}_{x}\right)+D_{w} \kappa^{2}\left(\left\{g_{1}^{2}+2 g_{1} g_{2}+g_{2}^{2}\right\}_{y}\right)\right] \tilde{\ddot{w}}^{2}\right. \\
& +L^{4}\left[2 D_{f}\left(\left\{f_{1}^{\prime \prime 2}+f_{2}^{\prime \prime 2}\right\}_{x}\right)+D_{w} \kappa^{2}\left(\left\{2 g_{1}^{\prime \prime 2}+g_{1}^{\prime \prime} g_{2}^{\prime \prime}+2 g_{2}^{\prime \prime 2}\right\}_{y}\right)\right] \tilde{w}^{2} \\
& +16 L^{2}(1-\nu)\left[D_{f}\left(\left\{f_{1}^{\prime 2}+f_{2}^{\prime 2}\right\}_{x}\right)+D_{w} \kappa^{2}\left(\left\{g_{1}^{\prime 2}+2 g_{1}^{\prime} g_{2}^{\prime}+g_{2}^{\prime 2}\right\}_{y}\right)\right] \tilde{\dot{w}}^{2} \\
& \left.+16 \nu L^{2}\left[D_{f}\left(\left\{f_{1}^{\prime \prime} f_{1}+f_{2}^{\prime \prime} f_{2}\right\}_{x}\right)+8 D_{w} \kappa^{2}\left(\left\{g_{1}^{\prime \prime} g_{1}+g_{2}^{\prime \prime} g_{2}+g_{1}^{\prime \prime} g_{2}+g_{1} g_{2}^{\prime \prime}\right\}_{y}\right)\right] \tilde{w} \tilde{\ddot{w}}\right\} \\
& +\frac{E L}{16}\left\{4\left(2 t_{f} b+t_{w} h\right)(\Delta-\tilde{\dot{u}})^{2}-4\left[t_{f}\left(\left\{f_{1}^{2}+f_{2}^{2}\right\}_{x}\right)+t_{w} \kappa^{2}\left(\left\{g_{1}^{2}+g_{2}^{2}\right\}_{y}\right)\right] \Delta \tilde{\dot{w}}^{2}\right. \\
& +4\left[t_{f}\left(\left\{f_{1}^{2}+f_{2}^{2}\right\}_{x}\right)+t_{w} \kappa^{2}\left(\left\{g_{1}^{2}+g_{2}^{2}\right\}_{y}\right)\right] \tilde{\dot{u}} \tilde{w}^{2} \\
& \left.+\left[t_{f}\left(\left\{f_{1}^{4}+f_{2}^{4}\right\}_{x}\right)+t_{w} \kappa^{4}\left(\left\{g_{1}^{4}+2 g_{1}^{2} g_{2}^{2}+g_{2}^{4}\right\}_{y}\right)\right] \tilde{\dot{w}}^{4}\right\}+\frac{G L^{3}}{16}\left\{t_{f}\left(\left\{f_{1}^{\prime 2} f_{1}^{2}+f_{2}^{\prime 2} f_{2}^{2}\right\}_{x}\right)\right. \\
& \left.+t_{w} \kappa^{4}\left(\left\{g_{1}^{\prime 2} g_{1}^{2}+2 g_{1}^{\prime} g_{1} g_{2}^{\prime} g_{2}+g_{2}^{\prime 2} g_{2}^{2}\right\}_{y}\right)\right\} \tilde{w}^{2} \tilde{w}^{2}-\frac{P L}{2}(\Delta-\tilde{\dot{u}}) \mathrm{d} \tilde{z} .
\end{aligned}
$$

Here, dots denote differentiation with respect to $\tilde{z}$ and primes denote differentiation with respect to $x$ for the function $f(x)$ and with respect to $y$ for the function $g(y)$. The braces with subscript $x$ denote that a definite integration has been performed on the function with respect to $x$ between the limits $x=[-b / 2, b / 2]$. Similarly, for the braces with subscript $y$, a definite integration has been performed with respect to $y$ between the limits $y=[-h, 0]$; for example:

$$
\left\{f_{1}^{\prime \prime} f_{1}\right\}_{x}=\int_{-b / 2}^{b / 2}\left\{\left[\frac{\mathrm{d}^{2}}{\mathrm{~d} x^{2}} f_{1}(x)\right] f_{1}(x)\right\} \mathrm{d} x, \quad\left\{g_{2}^{\prime \prime} g_{2}\right\}_{y}=\int_{-h}^{0}\left\{\left[\frac{\mathrm{d}^{2}}{\mathrm{~d} y^{2}} g_{2}(y)\right] g_{2}(y)\right\} \mathrm{d} y .
$$

The integrations are performed in the commercial computer algebra software MAPLE [29] and are not presented here in their explicit form for brevity. 


\subsection{Equilibrium equations}

The calculus of variations is performed on the total potential energy to obtain the governing equations of equilibrium for the case where local buckling only is present. The integrand of the total potential energy $V$ can be expressed as a Lagrangian [25], thus:

$$
V=\int_{0}^{2} \mathcal{L}(\tilde{\ddot{w}}, \tilde{\dot{w}}, \tilde{w}, \tilde{\dot{u}}, \tilde{u}, \tilde{z}) \mathrm{d} \tilde{z}
$$

and the first variation of $V$ is written thus:

$$
\delta V=\int_{0}^{2}\left\{\frac{\partial \mathcal{L}}{\partial \tilde{\ddot{w}}} \delta \tilde{\ddot{w}}+\frac{\partial \mathcal{L}}{\partial \tilde{\tilde{w}}} \delta \tilde{\dot{w}}+\frac{\partial \mathcal{L}}{\partial \tilde{w}} \delta \tilde{w}+\frac{\partial \mathcal{L}}{\partial \tilde{\dot{u}}} \delta \tilde{\dot{u}}+\frac{\partial \mathcal{L}}{\partial \tilde{u}} \delta \tilde{u}\right\} \mathrm{d} \tilde{z} .
$$

Since equilibrium is defined when the total potential energy is stationary, the first variation of $V$ must vanish for all small changes in $\tilde{w}$ and $\tilde{u}$. Since $\delta \tilde{\ddot{w}}=\mathrm{d}(\delta \tilde{\dot{w}}) / \mathrm{d} \tilde{z}, \delta \tilde{\dot{w}}=\mathrm{d}(\delta \tilde{w}) / \mathrm{d} \tilde{z}$ and $\delta \tilde{\dot{u}}=\mathrm{d}(\delta \tilde{u}) / \mathrm{d} \tilde{z}$, integration by parts allows the development of the Euler-Lagrange equations for $\tilde{w}$ and $\tilde{u}$; a fourth order nonlinear ordinary differential equation (ODE) in $\tilde{w}$ and a second order nonlinear ODE in $\tilde{u}$ are found respectively thus:

$$
\begin{aligned}
& \ddot{\ddot{w}}+\frac{L^{2}}{2 \eta}\left\{16 \nu\left[D_{f}\left(\left\{f_{1}^{\prime \prime} f_{1}+f_{2}^{\prime \prime} f_{2}\right\}_{x}\right)+D_{w} \kappa^{2}\left(\left\{g_{1}^{\prime \prime} g_{1}+g_{2}^{\prime \prime} g_{2}+g_{1}^{\prime \prime} g_{2}+g_{1} g_{2}^{\prime \prime}\right\}_{y}\right)\right] \tilde{\ddot{w}}\right. \\
& -16(1-\nu)\left[D_{f}\left(\left\{f_{1}^{\prime 2}+f_{2}^{\prime 2}\right\}_{x}\right)+D_{w} \kappa^{2}\left(\left\{g_{1}^{\prime 2}+g_{2}^{\prime 2}+2 g_{1}^{\prime} g_{2}^{\prime}\right\}_{y}\right)\right] \tilde{\ddot{w}} \\
& \left.+L^{2}\left[2 D_{f}\left(\left\{f_{1}^{\prime \prime 2}+f_{2}^{\prime \prime 2}\right\}_{x}\right)+D_{w} \kappa^{2}\left(2\left\{g_{1}^{\prime \prime 2}+2 g_{2}^{\prime \prime 2}+g_{1}^{\prime \prime} g_{2}^{\prime \prime}\right\}_{y}\right)\right] \tilde{w}\right\} \\
& -\frac{2 E L^{2}}{\eta}\left\{2\left[t_{f}\left(\left\{f_{1}^{2}+f_{2}^{2}\right\}_{x}\right)+t_{w} \kappa^{2}\left(\left\{g_{1}^{2}+g_{2}^{2}\right\}_{y}\right)\right](\tilde{\dot{u}} \tilde{\ddot{w}}+\tilde{\ddot{u}} \tilde{\dot{w}})+3\left[t_{f}\left(\left\{f_{1}^{4}+f_{2}^{4}\right\}_{x}\right)\right.\right. \\
& \left.\left.+t_{w} \kappa^{4}\left(\left\{g_{1}^{4}+2 g_{1}^{2} g_{2}^{2}+g_{2}^{4}\right\}_{y}\right)\right] \tilde{w}^{2} \tilde{\ddot{w}}-2\left[t_{f}\left(\left\{f_{1}^{2}+f_{2}^{2}\right\}_{x}\right)+t_{w} \kappa^{2}\left(\left\{g_{1}^{2}+g_{2}^{2}\right\}_{y}\right)\right] \Delta \tilde{\ddot{w}}\right\} \\
& -\frac{G L^{4}}{\eta}\left\{t_{f}\left(\left\{f_{1}^{\prime 2} f_{1}^{2}+f_{2}^{\prime 2} f_{2}^{2}\right\}_{x}\right)+t_{w} \kappa^{4}\left(\left\{g_{1}^{\prime 2} g_{1}^{2}+2 g_{1}^{\prime} g_{1} g_{2}^{\prime} g_{2}+g_{2}^{\prime 2} g_{2}^{2}\right\}_{y}\right)\right\}\left(\tilde{\ddot{w}} \tilde{w}^{2}+\tilde{\dot{w}}^{2} \tilde{w}\right)=0, \\
& \tilde{\ddot{u}}+\frac{\tilde{\dot{w}} \tilde{\ddot{w}}}{A}\left[t_{f}\left(\left\{f_{1}^{2}+f_{2}^{2}\right\}_{x}\right)+t_{w} \kappa^{2}\left(\left\{g_{1}^{2}+g_{2}^{2}\right\}_{y}\right)\right]=0,
\end{aligned}
$$

where the quantities $A$ and $\eta$ are defined:

$$
A=2 b t_{f}+h t_{w}, \quad \eta=16\left[D_{f}\left(\left\{f_{1}^{2}+f_{2}^{2}\right\}_{x}\right)+D_{w} \kappa^{2}\left(\left\{g_{1}^{2}+2 g_{1} g_{2}+g_{2}^{2}\right\}_{y}\right)\right],
$$

in which $A$ represents the total cross-sectional area of the strut and $\eta$ represents the relative bending and axial stiffnesses of the flange and the web. The total potential energy $V$ is also minimized with respect to the generalized coordinate $\Delta$, resulting in the integral equation:

$$
\int_{0}^{2}\left\{\tilde{\dot{u}}+\left[t_{f}\left(\left\{f_{1}^{2}+f_{2}^{2}\right\}_{x}\right)+t_{w} \kappa^{2}\left(\left\{g_{1}^{2}+g_{2}^{2}\right\}_{y}\right)\right] \frac{\tilde{\dot{w}}^{2}}{2 A}-\Delta+\frac{P}{E A}\right\} \mathrm{d} \tilde{z}=0 .
$$


Boundary conditions for $\tilde{w}$ and $\tilde{u}$ are also defined in order to find solutions for the set of equations above. The strut is assumed to be simply supported at $\tilde{z}=0$ and have symmetry conditions about the mid-span, at $\tilde{z}=1$. The boundary conditions can be written thus:

$$
\tilde{w}(0)=\tilde{\ddot{w}}(0)=\tilde{\dot{w}}(1)=\tilde{w}(1)=\tilde{u}(1)=0 .
$$

The symmetry condition is used to ensure that the solutions obtained are compatible with the later study on interactive buckling since the more severe cases occur when the global mode interacts with a symmetric local mode [22]. An additional end condition is also imposed at the point of loading, where $\tilde{z}=0$, to match the in-plane strain:

$$
\tilde{\dot{u}}+\left[t_{f}\left(\left\{f_{1}^{2}+f_{2}^{2}\right\}_{x}\right)+t_{w} \kappa^{2}\left(\left\{g_{1}^{2}+g_{2}^{2}\right\}_{y}\right)\right] \frac{\tilde{\dot{w}}^{2}}{2 A}-\Delta+\frac{P}{E A}=0,
$$

the expression arising naturally from minimizing $V$ given in Equation (25).

\subsection{Numerical results}

The governing equations (29)-(30) subject to equations (32)-(34) are solved numerically in the continuation and bifurcation software AUTO-07P [30] to calculate the local buckling load for an I-section strut with geometric and material properties detailed in Table 1. A

Table 1: Geometric and material properties of the strut in the numerical study.

\begin{tabular}{cc}
\hline Young's Modulus $E$ & $210000 \mathrm{~N} / \mathrm{mm}^{2}$ \\
Poisson's Ratio $\nu$ & 0.3 \\
Flange breadth $b$ & $90 \mathrm{~mm}$ \\
Flange thickness $t_{f}$ & $2.4 \mathrm{~mm}$ \\
Web thickness $t_{w}$ & $2.4 \mathrm{~mm}$ \\
Web height $h$ & $100 \mathrm{~mm}$ \\
\hline
\end{tabular}

range of lengths are selected for the strut that ensures local buckling remains critical. As discussed earlier, in previous work it was assumed that the flange to web out-of-plane displacement relationship parameter $\kappa$ was equal to $\kappa_{0}=-h /(\pi b)$, which is for the case where the flange-web joint is fully rigid. Using this assumption for $\kappa$, the critical loads for the local buckling mode are evaluated numerically using the analytical model and detailed in Table 2 in absolute terms and as a ratio of the global buckling load, as evaluated using the expression in Equation (22). The local buckling load of the flange for a long strut can be calculated by the well-known plate buckling expression:

$$
P_{l}^{\mathrm{C}}=\frac{k_{p} \pi^{2} E A}{12(1-\nu)^{2}\left(b_{f} / t_{f}\right)^{2}},
$$

where $A$ is the cross-sectional area of the strut defined in Equation (31), $b_{f}$ is the width of one half flange only and $k_{p}$ is the plate buckling coefficient for a rectangular simply supported long plate that is dependent upon the support conditions of the two unloaded edges 
Table 2: Critical loads of the local buckling mode for when $\kappa=\kappa_{0}$.

\begin{tabular}{cccccc}
\hline Length $[\mathrm{mm}]$ & 2000 & 2300 & 2500 & 2750 & 3000 \\
Critical load $P_{l}^{\mathrm{C}}[\mathrm{kN}]$ & 264.6 & 264.5 & 264.6 & 264.6 & 264.6 \\
Critical load ratio $P_{l}^{\mathrm{C}} / P_{o}^{\mathrm{C}}$ & 0.4128 & 0.5410 & 0.6370 & 0.7677 & 0.9110 \\
\hline
\end{tabular}

[31]. The vulnerable flange plates of all struts under consideration can be approximated as 'long' owing to a very high length to width ratio and it can be seen that the local buckling load for long plates is invariant with $L$, which agrees with Table 2. Using the loads calculated in Table 2, it can be deduced that for $\kappa=\kappa_{0}$, the $k_{p}$ value can be determined as 0.72 , which implies that the joint is stiffer than a completely pinned connection, where $k_{p}=0.43$, but considerably less stiff when compared to a rigid connection, where $k_{p}=1.25$; the reduction arising from the sympathetic web displacement during local buckling.

\subsection{FE model and validation}

In previous work [20], a strut of identical material and geometric properties was constructed in the commercial finite element package ABAQUS [21] to validate the perfect case strut [20]. A similar model is used presently to validate the numerical results from the analytical model for the strut where only local buckling is present. Quadrilateral four-noded linear shell elements (S4R) are used with reduced integration and hourglass control is applied to avoid unphysical solutions. The components of the strut are created in separate parts and are tied together using the 'No Rotation' constraint. An eigenvalue analysis is used to find the local buckling load. A model is constructed for each strut length under consideration and analysed in basically the same way for each case.

\subsubsection{Calibration of $\kappa$}

The lowest local buckling loads found in ABAQUS are shown in Fig. 6, along with the numerical results from the analytical model, for the case when $\kappa=\kappa_{0}$. It can be seen that the FE model predicts a considerably lower local buckling load than the analytical model. This is in fact a predictable result since approximations are used to model shape functions $f_{i}(x)$ and $g_{i}(y)$ and so the result would theoretically be an upper bound to the true solution. The parameter $\kappa$ is therefore also an instrument to reduce the overall errors arising from introducing the approximations. The characteristic stiffness of the flange-web joint is one of the key parameters determining the local buckling load and the difference in the analytical and FE predictions indicates that the former overestimates the overall rigidity of the flange--web joint. Hence, $\kappa$ is reduced in magnitude such that it represents the actual connection stiffness better and thereby matches the local buckling loads.

It is found that the best matches are achieved when $\kappa=0.65 \kappa_{0} \equiv \kappa_{c}$. The numerical results found using the new $\kappa$ value are also shown in Fig. 6 ; it is evident that the calibrated value of $\kappa=\kappa_{c}$ results in a much more accurate measure of the local buckling load for all lengths considered. Using the expression defined in Equation (35) it can be calculated for 


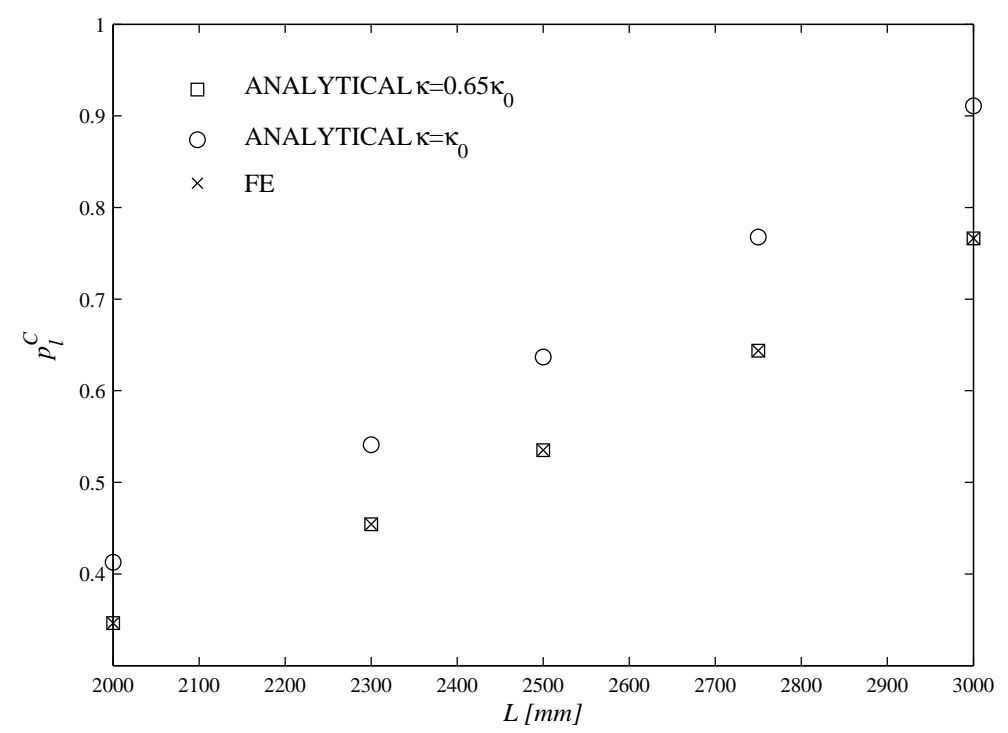

Figure 6: Normalized local buckling loads $p_{l}^{\mathrm{C}}\left(=P_{l}^{\mathrm{C}} / P_{o}^{\mathrm{C}}\right)$ for a range of strut lengths, with analytical results denoted using circles $\left(\kappa=\kappa_{0}\right)$ and squares $\left(\kappa=0.65 \kappa_{0} \equiv \kappa_{c}\right)$ and the FE results are denoted with crosses.

$\kappa=\kappa_{c}$ value that the plate buckling coefficient $k_{p}$ is now 0.62 , confirming a considerably less rigid flange--web connection than determined previously.

\section{Nonlinear model for interactive buckling with geometric imperfections}

The primary purpose of the current article is to conduct an imperfection sensitivity study for long I-section struts, where global buckling is critical but subsequently interacts with the local buckling mode. The general form of the energy equations detailed in Equations (9)-(19), are developed to generate the total potential energy such that interactive buckling is modelled. For this case, one flange is less compressed than the other, owing to the global buckling of the strut. It is assumed that no out-of-plane deflection occurs in the less compressed flange. This is primarily to simplify the formulation but also noting that in previous work [32] it had been demonstrated that there may be insignificant influence from the less compressed parts of the cross-section on the nonlinear behaviour when global buckling is critical. This implies that in the total potential energy, all deflection functions with a subscript "2", as well as the associated initial imperfections, namely $u_{f 2}(z)=u_{w 2}(z)=w_{f 2}(z)=w_{w 2}(z)=w_{f 20}(z)=w_{w 20}(z)=0$. The notation is therefore simplified thus: $w_{f 1}(z) \equiv w(z), u_{f 1}(z) \equiv u(z), f_{1}(x) \equiv f(x), g_{1}(y) \equiv g(y)$ and $w_{f 0} \equiv w_{0}$. It is also worth noting that the $\kappa$ parameter has been implemented once again, where $w_{w}(z)=\kappa w(z)$ and $w_{w 0}(z)=\kappa w_{0}(z)$. Hence, the total potential energy can be written 
thus:

$$
\begin{aligned}
& V=\int_{0}^{2}\left\{\frac{E I_{f}\left(q_{s}-q_{s 0}\right)^{2} \pi^{4}}{2 L} \sin ^{2}\left(\frac{\pi \tilde{z}}{2}\right)+\frac{1}{16 L}\left\{16\left(D_{f}\left\{f^{2}\right\}_{x}+D_{w} \kappa^{2}\left\{g^{2}\right\}_{y}\right)\left(\tilde{\ddot{w}}-\tilde{\ddot{w}}_{0}\right)^{2}\right.\right. \\
& +8 L^{2}\left[\nu\left(D_{f}\left\{f^{\prime \prime} f\right\}_{x}+D_{w} \kappa^{2}\left\{g^{\prime \prime} g\right\}_{y}\right)\left(\tilde{\ddot{w}}-\tilde{\ddot{w}}_{0}\right)\left(\tilde{w}-\tilde{w}_{0}\right)+(1-\nu)\left(D_{f}\left\{f^{\prime 2}\right\}_{x}\right.\right. \\
& \left.\left.\left.+D_{w} \kappa^{2}\left\{g^{\prime 2}\right\}_{y}\right)\left(\tilde{\dot{w}}-\tilde{\dot{w}}_{0}\right)^{2}\right]+L^{4}\left(D_{f}\left\{f^{\prime \prime 2}\right\}_{x}+D_{w} \kappa^{2}\left\{g^{\prime \prime 2}\right\}_{y}\right)\left(\tilde{w}-\tilde{w}_{0}\right)^{2}\right\} \\
& +\frac{E L}{4}\left[\left(6 b t_{f} b+h t_{w}\right) \frac{\left(q_{t}-q_{t 0}\right)^{2} h^{2} \pi^{4}}{12 L^{2}} \sin ^{2}\left(\frac{\pi \tilde{z}}{2}\right)+\left(2 b t_{f}+h t_{w}\right) \Delta(\Delta-\tilde{\dot{u}})\right. \\
& -\left(\left\{f^{2}\right\}_{x} t_{f}+t_{w} \kappa^{2}\left\{g^{2}\right\}_{y}\right) \Delta\left(\tilde{\dot{w}}^{2}-\tilde{\dot{w}}_{0}^{2}\right)+\left(b t_{f}+\frac{h t_{w}}{3}\right) \tilde{\dot{u}}^{2} \\
& +\left(t_{f}\left\{f^{2}\right\}_{x}-\frac{t_{w} \kappa^{2}\left\{y g^{2}\right\}_{y}}{h}\right) \tilde{\dot{u}}\left(\tilde{\dot{w}}^{2}-\tilde{\dot{w}}_{0}^{2}\right)+\frac{\left(t_{f}\left\{f^{4}\right\}_{x}+t_{w} \kappa^{4}\left\{g^{4}\right\}_{y}\right)}{4}\left(\tilde{\dot{w}}^{2}-\tilde{\dot{w}}_{0}^{2}\right)^{2} \\
& -\frac{\left(q_{t}-q_{t 0}\right) \pi^{2}}{L}\left\{\left[\frac{t_{f}\left\{f^{2}\right\}_{x} h}{2}-t_{w} \kappa^{2}\left(\left\{y g^{2}\right\}_{y}+\frac{h\left\{g^{2}\right\}_{y}}{2}\right)\right]\left(\tilde{\dot{w}}^{2}-\tilde{\dot{w}}_{0}^{2}\right)\right. \\
& \left.\left.+\left(b h t_{f}+\frac{h^{2} t_{w}}{6}\right) \tilde{\dot{u}}\right\} \sin \left(\frac{\pi \tilde{z}}{2}\right)\right]+\frac{G L^{3} t_{w}}{16 h}\left[4\left(q_{s}-q_{s 0}-q_{t}+q_{t 0}\right)^{2} \frac{\pi^{2} h^{2}}{L^{2}} \cos ^{2}\left(\frac{\pi \tilde{z}}{2}\right)\right. \\
& +\tilde{u}^{2}+h\left(\frac{t_{f}}{t_{w}}\left\{f^{\prime 2} f^{2}\right\}_{x}+\kappa^{4}\left\{g^{\prime 2} g^{2}\right\}_{y}\right)\left(\tilde{w} \tilde{\dot{w}}-\tilde{w}_{0} \tilde{\dot{w}}_{0}\right)^{2}-2 \kappa^{2}\left\{g^{\prime} g\right\}_{y} \tilde{u}\left(\tilde{w} \tilde{\dot{w}}-\tilde{w}_{0} \tilde{\dot{w}}_{0}\right) \\
& \left.-4\left(q_{s}-q_{s 0}-q_{t}+q_{t 0}\right) \frac{\pi h}{L}\left[\tilde{u}-\kappa^{2}\left\{g^{\prime} g\right\}_{y}\left(\tilde{w} \tilde{\dot{w}}-\tilde{w}_{0} \tilde{\dot{w}}_{0}\right)\right] \cos \left(\frac{\pi \tilde{z}}{2}\right)\right] \\
& \left.-\frac{P L}{2}\left[\frac{q_{s}^{2} \pi^{2}}{2} \cos ^{2}\left(\frac{\pi \tilde{z}}{2}\right)-\frac{1}{2} \tilde{\dot{u}}+\Delta\right]\right\} \mathrm{d} \tilde{z} .
\end{aligned}
$$

\subsection{Governing equations}

The calculus of variations is performed, as detailed earlier, on the total potential energy to obtain the governing equations of equilibrium for the case of a long strut with initial geometric imperfections. The resulting fourth order nonlinear ODE in $\tilde{w}$ and second order nonlinear ODE in $\tilde{u}$ respectively are as follows:

$$
\begin{aligned}
& \left(\ddot{\ddot{w}}-\ddot{\ddot{w}}_{0}\right)+\frac{L^{2}}{\eta}\left\{L^{2}\left(\tilde{w}-\tilde{w}_{0}\right)\left[D_{f}\left\{f^{\prime \prime 2}\right\}_{x}+D_{w} \kappa^{2}\left\{g^{\prime \prime 2}\right\}_{y}\right]\right. \\
& \left.\quad+8\left(\tilde{\ddot{w}}-\tilde{\ddot{w}}_{0}\right)\left[D_{f}\left(\nu\left\{f^{\prime \prime} f+f^{\prime 2}\right\}_{x}-\left\{f^{\prime 2}\right\}_{x}\right)+D_{w} \kappa^{2}\left(\nu\left\{g^{\prime \prime} g+g^{\prime 2}\right\}_{y}-\left\{g^{\prime 2}\right\}_{y}\right)\right]\right\} \\
& \quad-\frac{2 E L^{2}}{\eta}\left\{\left(3 \tilde{w}^{2} \tilde{\ddot{w}}-\tilde{\ddot{w}} \tilde{w}_{0}^{2}-2 \tilde{w}_{\tilde{w}_{0}} \tilde{\ddot{w}}_{0}\right)\left[t_{f}\left\{f^{4}\right\}_{x}+t_{w} \kappa^{4}\left\{g^{4}\right\}_{y}\right]\right. \\
& \quad+\frac{\left(q_{t}-q_{t 0}\right) h \pi^{2}}{L}\left[t_{w} \kappa^{2}\left(\frac{2}{h}\left\{y g^{2}\right\}_{y}+\left\{g^{2}\right\}_{y}\right)-t_{f}\left\{f^{2}\right\}_{x}\right]\left[\sin \left(\frac{\pi \tilde{z}}{2}\right) \tilde{\ddot{w}}+\frac{\pi}{2} \cos \left(\frac{\pi \tilde{z}}{2}\right) \tilde{\dot{w}}\right] \\
& \left.\quad+2(\tilde{\ddot{u}} \tilde{\dot{w}}+\tilde{\dot{u}} \tilde{\ddot{w}})\left(t_{f}\left\{f^{2}\right\}_{x}-\frac{t_{w} \kappa^{2}\left\{y g^{2}\right\}_{y}}{h}\right)-2 \Delta \tilde{\ddot{w}}\left(t_{f}\left\{f^{2}\right\}_{x}+t_{w} \kappa^{2}\left\{g^{2}\right\}_{y}\right)\right\}
\end{aligned}
$$




$$
\begin{aligned}
& -\frac{G L^{4}}{\eta}\left\{\tilde{w}\left(\tilde{w} \tilde{\ddot{w}}+\tilde{w}^{2}-\tilde{w}_{0}^{2}-\tilde{w}_{0} \tilde{\ddot{w}}_{0}\right)\left(t_{f}\left\{f^{\prime 2} f^{2}\right\}_{x}+t_{w} \kappa^{4}\left\{g^{\prime 2} g^{2}\right\}_{y}\right)\right. \\
& \left.-\frac{t_{w} \kappa^{2}\left\{g^{\prime} g\right\}_{y}}{h} \tilde{w}\left[\tilde{\dot{u}}+\left(q_{s}-q_{s 0}-q_{t}+q_{t 0}\right) \frac{h \pi^{2}}{L} \sin \left(\frac{\pi \tilde{z}}{2}\right)\right]\right\}=0, \\
\tilde{\ddot{u}}- & \frac{G L^{2} t_{w}}{2 E h \zeta}\left[\tilde{u}-\frac{2 h \pi\left(q_{s}-q_{s 0}-q_{t}+q_{t 0}\right)}{L} \cos \left(\frac{\pi \tilde{z}}{2}\right)-\kappa^{2}\left\{g^{\prime} g\right\}_{y}\left(\tilde{w} \tilde{\dot{w}}-\tilde{w}_{0} \tilde{\dot{w}}_{0}\right)\right] \\
- & \frac{\left(q_{t}-q_{t 0}\right) h \pi^{3}}{2 L \zeta}\left[b t_{f}+\frac{h t_{w}}{6}\right] \cos \left(\frac{\pi \tilde{z}}{2}\right)+\frac{2}{\zeta}\left[t_{f}\left\{f^{2}\right\}_{x}-\frac{t_{w} \kappa^{2}}{h}\left\{y g^{2}\right\}_{y}\right]\left(\tilde{\tilde{w}} \tilde{\dot{w}}-\tilde{\tilde{w}}_{0} \tilde{\dot{w}}_{0}\right)=0,
\end{aligned}
$$

where $\zeta$ is defined and $\eta$ is updated respectively thus:

$$
\zeta=2 t_{f} b+\frac{2}{3} t_{w} h, \quad \eta=16\left[D_{f}\left\{f^{2}\right\}_{x}+D_{w} \kappa^{2}\left\{g^{2}\right\}_{y}\right],
$$

The total potential energy $V$ is also minimized with respect to the generalized coordinates $q_{s}, q_{t}$ and $\Delta$, thus:

$$
\begin{gathered}
\left(q_{s}-q_{s 0}\right) \pi^{2}+\frac{G h L^{2} t_{w}}{2 E I_{f}}\left(q_{s}-q_{s 0}-q_{t}+q_{t 0}\right)-\frac{P L^{2}}{2 E I_{f}} q_{s} \\
+\frac{G L^{3} t_{w}}{4 E I_{f} \pi} \int_{0}^{2}\left[\kappa^{2}\left\{g^{\prime} g\right\}_{y}\left(\tilde{\dot{w}} \tilde{w}-\tilde{\dot{w}}_{0} \tilde{w}_{0}\right)-\tilde{u}\right] \cos \left(\frac{\pi \tilde{z}}{2}\right) \mathrm{d} \tilde{z}=0, \\
\left(q_{t}-q_{t 0}\right) \pi^{2}\left(1+\frac{h t_{w}}{6 b t_{f}}\right)-\frac{2 G t_{w} L^{2}}{E b h t_{f}}\left(q_{s}-q_{s 0}-q_{t}+q_{t 0}\right)+\int_{0}^{2}\left\{\frac { L t _ { w } } { b t _ { f } } \left[\left(\frac{\left\{y g^{2}\right\}_{y}}{h^{2}}\right.\right.\right. \\
\left.\left.+\frac{\kappa^{2}\left\{g^{2}\right\}_{y}}{2 h}\right)\left(\tilde{\dot{w}}^{2}-\tilde{\dot{w}}_{0}^{2}\right)-\frac{1}{6} \tilde{\dot{u}}\right] \sin \left(\frac{\pi \tilde{z}}{2}\right)-\frac{L}{h}\left[\tilde{\dot{u}}+\frac{\left\{f^{2}\right\}_{x}}{2 b}\left(\tilde{\dot{w}}^{2}-\tilde{\dot{w}}_{0}^{2}\right)\right] \sin \left(\frac{\pi \tilde{z}}{2}\right) \\
\left.\quad+\frac{G L^{3} t_{w}}{E b h^{2} t_{f} \pi}\left[\tilde{u}-\kappa^{2}\left\{g^{\prime} g\right\}_{y}\left(\tilde{w} \tilde{\dot{w}}-\tilde{w}_{0} \tilde{\dot{w}}_{0}\right)\right] \cos \left(\frac{\pi \tilde{z}}{2}\right)\right\} \mathrm{d} \tilde{z}=0, \\
\int_{0}^{2}\left\{\Delta-\frac{1}{2} \tilde{\dot{u}}^{2}\left[t_{f}\left\{f^{2}\right\}_{x}+t_{w} \kappa^{2}\left\{g^{2}\right\}_{y}\right] \frac{\left(\tilde{\dot{w}}^{2}-\tilde{\dot{w}}_{0}^{2}\right)}{2 A}-\frac{P}{E A}\right\} \mathrm{d} \tilde{z}=0 .
\end{gathered}
$$

Using Equation (41), a relationship can be established between the two global imperfections $q_{s 0}$ and $q_{t 0}$ before any interactive buckling occurs, which reduces the number of global imperfection parameters to one:

$$
q_{s 0}=\left[1+\pi^{2}\left(1+\frac{h t_{w}}{6 b t_{f}}\right)\left(\frac{E b h t_{f}}{2 G L^{2} t_{w}}\right)\right] q_{t 0} .
$$

Boundary conditions for $\tilde{w}$ and $\tilde{u}$ are also defined in the same way as presented in Equations (33)-(34), the former is precisely the same, but the latter condition, which is evaluated at $\tilde{z}=0$, changes thus:

$$
2 b t_{f} \tilde{\dot{u}}\left(1+\frac{h t_{w}}{3 b t_{f}}\right)-A \Delta+\left(\tilde{\dot{w}}^{2}-\tilde{\dot{w}}_{0}^{2}\right)\left(t_{f}\left\{f^{2}\right\}_{x}-\frac{t_{w} \kappa^{2}}{h}\left\{y g^{2}\right\}_{y}\right)+\frac{P}{E}=0 .
$$


Note that the global buckling load $P_{o}^{\mathrm{C}}$ can also be determined in the same way as described earlier, resulting in the same expression as presented in Equation (22).

\section{Numerical results from the interactive buckling model}

\subsection{Perfect case}

The calibrated coefficient, $\kappa=\kappa_{c}$, determined in $\S 3.3 .1$, is now incorporated into the perfect case analytical model for interactive buckling. The solution for this problem for the case where $\kappa=\kappa_{0}$ has been presented in the authors' previous work [20]. It is anticipated that with the local buckling load prediction being improved, a commensurate improvement in the comparisons between the calibrated analytical model and the FE model will also be observed in the nonlinear range.

For the perfect case, it is assumed that $q_{s 0}=q_{t 0}=w_{0}=0$ in the governing equations, which are solved for the geometric and material properties detailed in Table 1, with a length of $4000 \mathrm{~mm}$. The geometries are selected such that global strong axis buckling is the critical mode, followed by a secondary bifurcation that triggers mode interaction with local plate buckling shortly afterwards. The nonlinear equilibrium equations are solved using the continuation and bifurcation software AUTO-07P and then validated with a nonlinear FE model developed in ABAQUs using the Riks analysis procedure [33]. The solution strategy for the analytical model is fundamentally the same as that in earlier related work [24]. Figure 7 shows sketches of examples of perfect and imperfect paths for
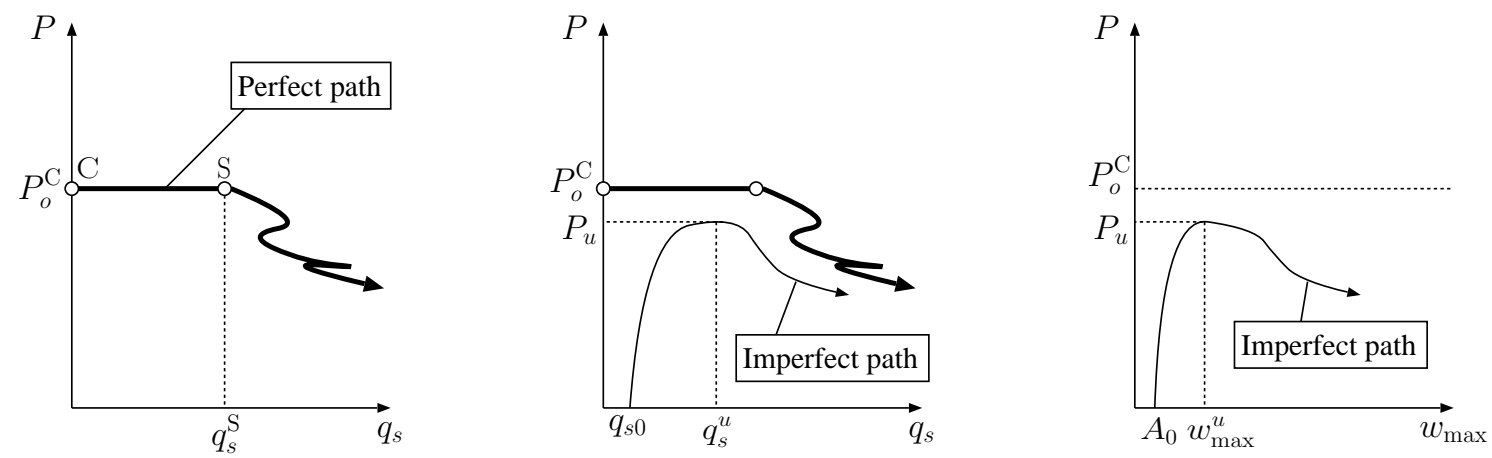

Figure 7: Sketches of equilibrium diagrams for the global critical buckling case with a combined global and local imperfection: (left) initially weakly stable (flat) perfect post-buckling path shown with critical and secondary bifurcations $\mathrm{C}$ and $\mathrm{S}$; (middle and right) imperfect path for load $P$ versus $q_{s}$ and $w_{\max }$ respectively.

struts where global buckling is critical. The critical and secondary bifurcations $\mathrm{C}$ and $\mathrm{S}$ respectively, the ultimate load of the imperfect systems $P_{u}$ and the corresponding values of $q_{s}^{\mathrm{u}}$ and $w_{\max }^{\mathrm{u}}$ that define the load carrying capacity and corresponding deflection levels of an imperfect strut are highlighted.

For the FE model, the imperfections are kept to be as small as possible, with the global imperfection set at $L / 20000$ and the local imperfection at $t_{f} / 150$, such that the response is compared relatively fairly with the perfect case analytical model. The post-buckling 

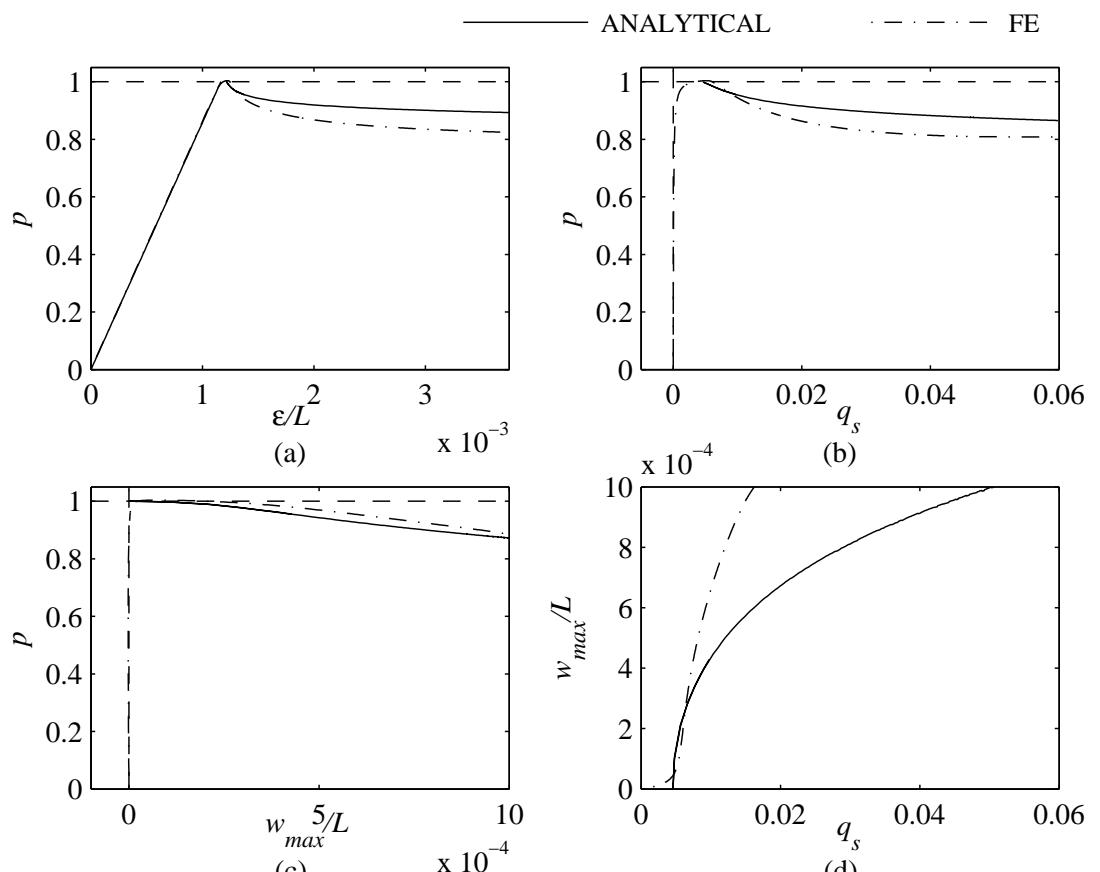

(c)

(d)

Figure 8: Numerical solutions of the equilibrium equations from the analytical model (solid line) and FE solutions (dot-dashed line) for the perfect strut where $\kappa=\kappa_{c}$. Graphs show (a) the normalized load $p=P / P_{o}^{\mathrm{C}}$ versus the normalized total end-shortening $\mathcal{E} / L$, (b) $p$ versus the global mode amplitude $q_{s}$, (c) $p$ versus the maximum out-of-plane flange deflection $w_{f, \max } / L$ and (d) $w_{f, \max } / L$ versus $q_{s}$.

paths found using both methods are shown in Fig. 8. A very good comparison of the paths can be observed from the figures, particularly in the neighbourhood of the secondary bifurcation predicted by the analytical model. Compared to the previous results for a fully rigid flange-web joint [20], in particular, the global buckling displacement versus the out-of-plane displacement shown in Fig. 8(d) is improved.

This comparison can be observed in greater depth by examining Figs. 9 and 10, where the out-of-plane displacement profiles of the flange and the web respectively are shown at different stages of the post-buckling response. Immediately after the secondary bifurcation point, i.e. when $p=0.998$, the comparisons between the two models are excellent, with the out-of-plane displacement profile of both the flange and the web appearing to be almost identical, a considerable improvement on the previous comparison for when $\kappa=\kappa_{0}$, as presented in [20]. For the previous case, the maximum amplitude of the out-of-plane displacement profile was similar but the wavelengths predicted by the analytical model were initially visibly shorter. With the calibrated parameter of $\kappa=\kappa_{c}$, the joint rotational stiffness is smaller hence the wavelengths found in the out-of-plane displacement profiles currently are initially longer, which improves the comparison with the FE model; this agrees qualitatively with earlier work on I-section struts buckling globally about the weak axis [32]. The out-of-plane displacement of the web is also shown in Fig. 10, which is 


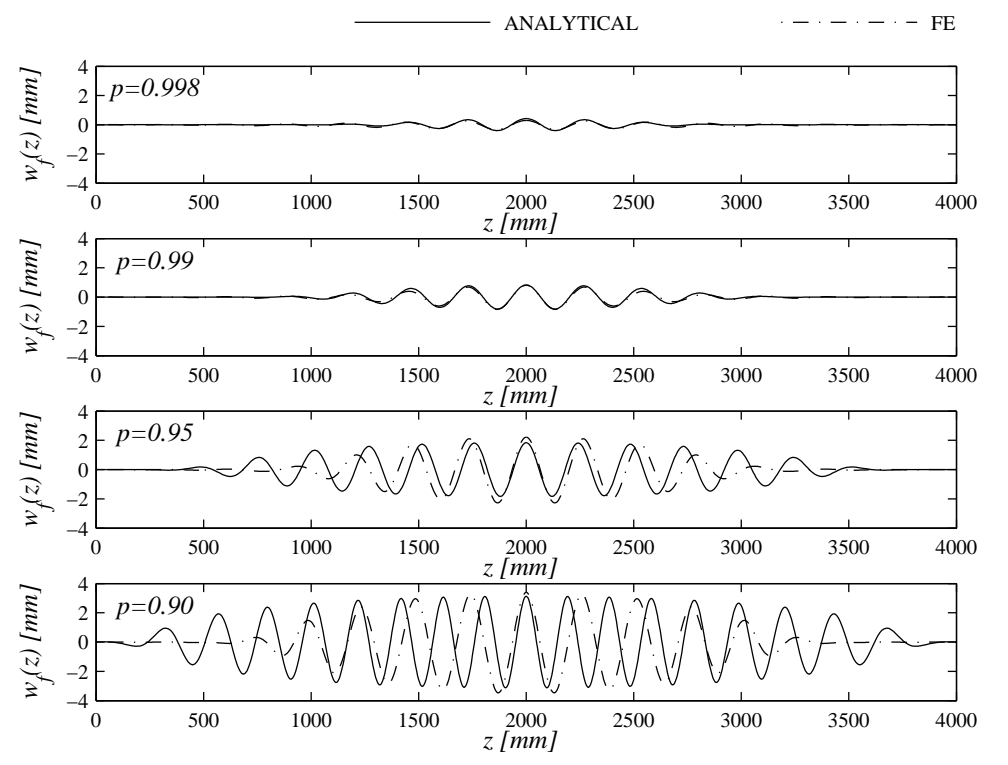

Figure 9: Comparisons for the perfect case strut of the out-of-plane displacement function $w_{f}$ of the flange between the numerical solution of the analytical model (solid line) and the FE model (dot-dashed line), for different normalized loads $p$ in the post-buckling range.

calculated in the analytical model by multiplying the deflection of the flange by $\kappa_{c}$. It can be observed that, again, there is an excellent comparison between the analytical and the FE models, indicating that the web deflection has also been captured successfully by the analytical model using the calibrated $\kappa$ value.

As the post-buckling path progresses, the solutions for the analytical and FE models begin to diverge, which can be seen in both the equilibrium paths as well as the outof-plane flange and web displacement profiles. The analytical model exhibits a slightly stiffer post-buckling response, which can be attributed partially to the rapidly decreasing wavelengths in the out-of-plane displacement profile, as the load applied is influenced by the frequency of the waves present [34]. The wavelength of the out-of-plane displacement profile predicted by the $\mathrm{FE}$ model changes at a much slower rate, which results in a less stiff response in the post-buckling range. The phenomenon of significantly decreasing wavelengths has been observed experimentally in similar components [12, 35, 16, 36] and it has also been demonstrated in previous work that static FE models struggle to model this phenomena successfully [13, 17]. Thus, the analytical model perhaps may be said to give a more accurate representation of the physical behaviour of the system in terms of the local buckling wavelength.

The progression of the deflections in the entire cross-section at the strut midspan is shown in Fig. 11 at different stages in the post-buckling process. Note that deflections in this diagram have been exaggerated by a factor of 5 for clarity. Again, an excellent comparison is observed in the first figure, immediately after the secondary bifurcation 

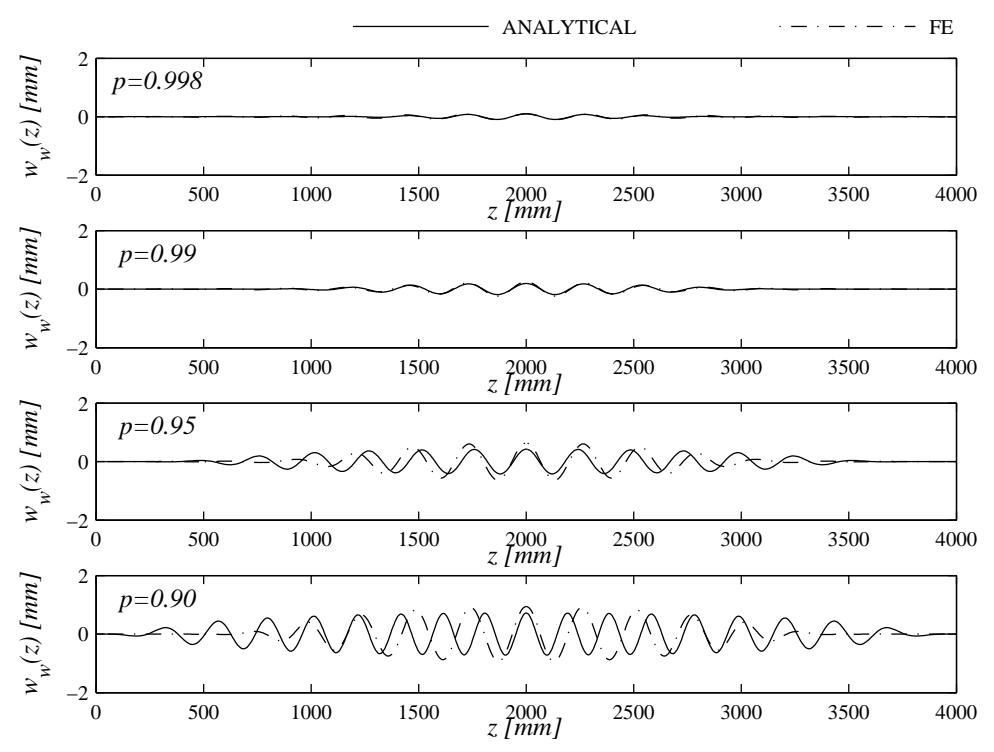

Figure 10: Comparisons for the perfect case strut of the out-of-plane displacement function $w_{w}$ of the web between the numerical solution of the analytical model (solid line) and the FE model (dot-dashed line), for different normalized loads $p$ in the post-buckling range.

point. As the post-buckling path progresses, however, the comparisons begin to diverge slightly, most notably in the less compressed top flange. Here, the FE model shows some out-of-plane deflections, whereas in the analytical model the corresponding deflection in the less compressed flange is assumed to be zero. From the cross-section diagrams, it can be seen that this is arguably a reasonable assumption especially in the initial post-buckling range, when compared to the magnitude of deflections in the more compressed flange or the web. However, the displacement is clearly not equal to zero due to the transfer of stress between the joints of the flanges to the web. This is another, perhaps stronger, contributory factor explaining the less stiff response exhibited by the FE model and the divergence of the paths in the advanced post-buckling range.

\subsection{Struts with initial imperfections}

The governing equations including the initial imperfections are now solved numerically with AUTO-07P using the geometries selected for the strut shown in Table 1 with a length of $4000 \mathrm{~mm}$. The current work investigates the effects of the global imperfection for the calibrated model, where $\kappa=\kappa_{c}$, as well as the effects of different local imperfections on the strut for a variety of displacement profiles and magnitudes. Finally, the strut is analysed for the cases where both global out-of-straightness and local imperfections are present simultaneously. 


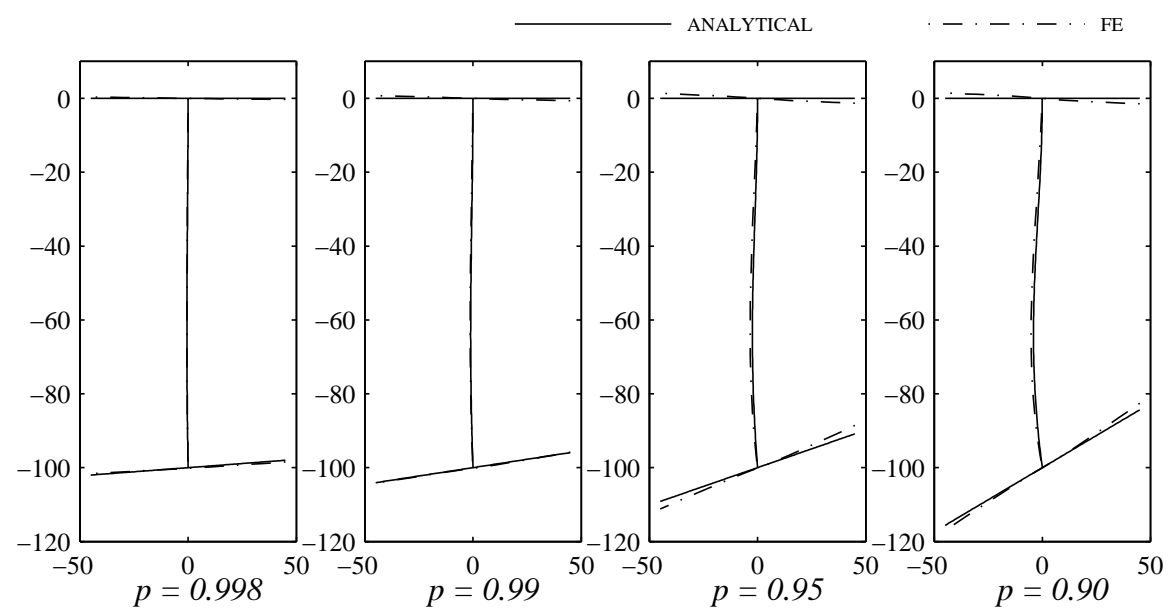

Figure 11: Out-of-plane cross-sectional deflections of the I-section strut at midspan, shown for four separate loads on the post-buckling path. All deflections are scaled by a factor of 5 to aid clarity and each graph shows the $x$ and $y$ axes in millimetres, as defined in Fig. 1.

\subsubsection{Global imperfections only: $q_{s 0}, q_{t 0} \neq 0, w_{0}=0$}

To investigate the sensitivity of the strut to global imperfections, the normalized initial global deflection $q_{s 0}$ (alongside the corresponding $q_{t 0}$ term) is increased parametrically from the perfect case solution in the software Auto-07P, ranging from 1/10000 to $1 / 350$. The study has already been conducted in previous work for the case where the flange-web joint is fully rigid, with $\kappa=\kappa_{0}$ [20]. The study is only mentioned currently for completeness, with the application of the calibrated $\kappa=\kappa_{c}$ value, yielding qualitatively similar results. The ultimate loads found for each magnitude of initial imperfection are slightly lower than for the fully rigid joint case, but do not differ by more than $3 \%$, for all magnitudes of global imperfections analysed.

\subsubsection{Local imperfections only: $q_{s 0}, q_{t 0}=0, w_{0} \neq 0$}

For the study where only local imperfections are present, the out-of-straightness parameters $q_{s 0}$ and $q_{t 0}$ are both set to zero in the governing equations. The initial local imperfection is introduced to the more compressed flange as well as the web, and the set of governing equations is solved for a selection of $A_{0}, \alpha$ and $\beta$ values for all imperfections (with $A_{1}=\kappa_{c} A_{0}$ for the web plate). A consistent method for determining the size of the imperfection must be defined for all variations of the local imperfection profile. Thus, the size of the local imperfection can be found by the expression:

$$
\mathcal{E}_{0}=\frac{1}{2} \int_{0}^{L} \dot{w}_{0}^{2} \mathrm{~d} z
$$

which gives the total end-shortening due to the deflected shape of the imperfection. Parameters $A_{0}, \alpha$ and $\beta$ are varied systematically from the perfect case solution [20] to study the effect of the different local imperfections at distinct stages. 
First, only $\beta$ is varied, which must take on odd integer values to satisfy the symmetry boundary condition at midspan, while $\alpha$ is kept fixed at zero. The resulting imperfection shape is therefore purely periodic, with no modulation (or localization) in the amplitude. The initial end-shortening parameter $\mathcal{E}_{0}$ is then increased to preset magnitudes and the equilibrium path for a range of $\beta$ and corresponding $A_{0}$ values are recorded for each imperfection size. The $\beta$ values and corresponding amplitudes $A_{0}$ that give the lowest ultimate load for each size of imperfection are recorded and plotted in Fig. 12(a).

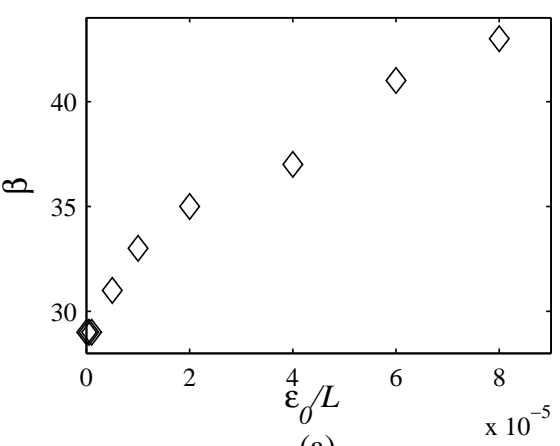

(a)

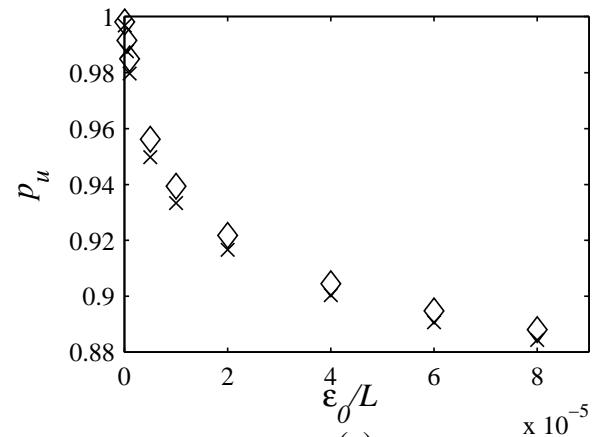

(c)

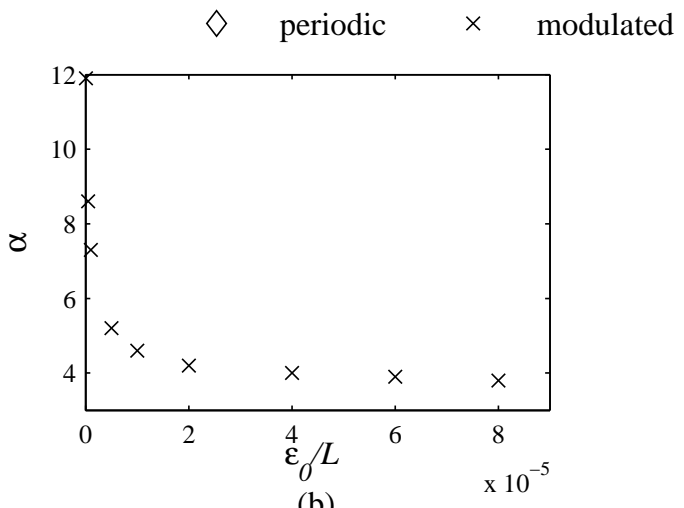

(b)

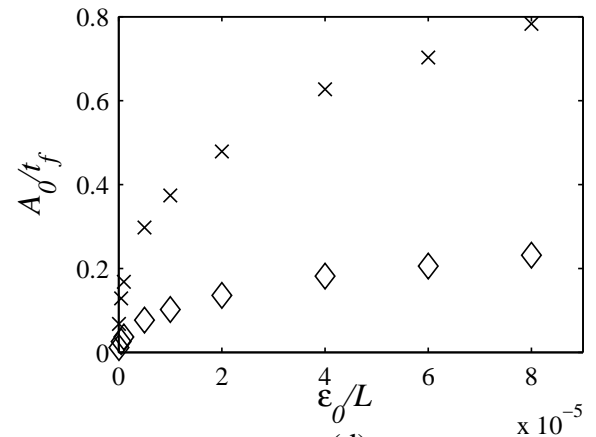

(d)

Figure 12: Imperfection sensitivity diagrams for both periodic imperfection shapes (diamonds) and modulated imperfection shapes (crosses). All graphs are plotted with the normalized imperfection size $\mathcal{E}_{0} / L$ on the abscissa. Graph (a) shows the $\beta$ value that gives the lowest ultimate load found with a periodic imperfection; (b) shows the $\alpha$ value for each worst case $\beta$ value that gives lowest ultimate load for a modulated imperfection; (c) shows the normalized ultimate loads $p_{u}=P_{u} / P_{o}^{\mathrm{C}}$ found and (d) shows the imperfection midspan normalized amplitude $A_{0} / t_{f}$.

In the second investigation, a modulated local imperfection shape is introduced $(\alpha \neq 0)$. The initial end-shortening $\mathcal{E}_{0}$ and parameter $\beta$ are kept constant, where $\beta$ is taken as the value that gives the lowest ultimate load for a purely periodic imperfection in the previous stage. Then $\alpha$ is increased and $A_{0}$ is adjusted for the given $\mathcal{E}_{0}$ value. The combination of $\alpha$ and $A_{0}$ that give the lowest ultimate load for each $\mathcal{E}_{0}$ value is also recorded and plotted in Fig. 12(b). The ultimate loads for purely periodic as well as modulated imperfections are also included diagrammatically in Fig. 12(c) with the distribution of amplitudes versus the imperfection size shown in Fig. 12(d). 
For purely periodic functions, the ultimate load gradually decreases as $\mathcal{E}_{0}$ increases, as expected. The periodicity parameter $\beta$ varies for each size of imperfection as observed in Fig. 12(a), increasing slowly with $\mathcal{E}_{0}$. A further reduction in ultimate load is observed with the introduction of the modulated imperfection shape (i.e. $\alpha \neq 0$ ), although the effect is subtle with a less than $1 \%$ decrease for all sizes of imperfection. As demonstrated in Fig. 12(b), a smaller amount of localization is required to give the lowest ultimate load for larger imperfections and $\alpha$ converges asymptotically to a value of approximately 4 for larger initial imperfection sizes in the current example geometry.

The strut shows sensitivity to both types of imperfections. The reduction in ultimate load for the largest initial end-shortening $\mathcal{E}_{0}$ considered is approximately $11.3 \%$ for the periodic imperfection and $11.6 \%$ for the modulated imperfection when compared to the perfect case. Figure 13 shows the family of equilibrium paths with the worst case combinations of
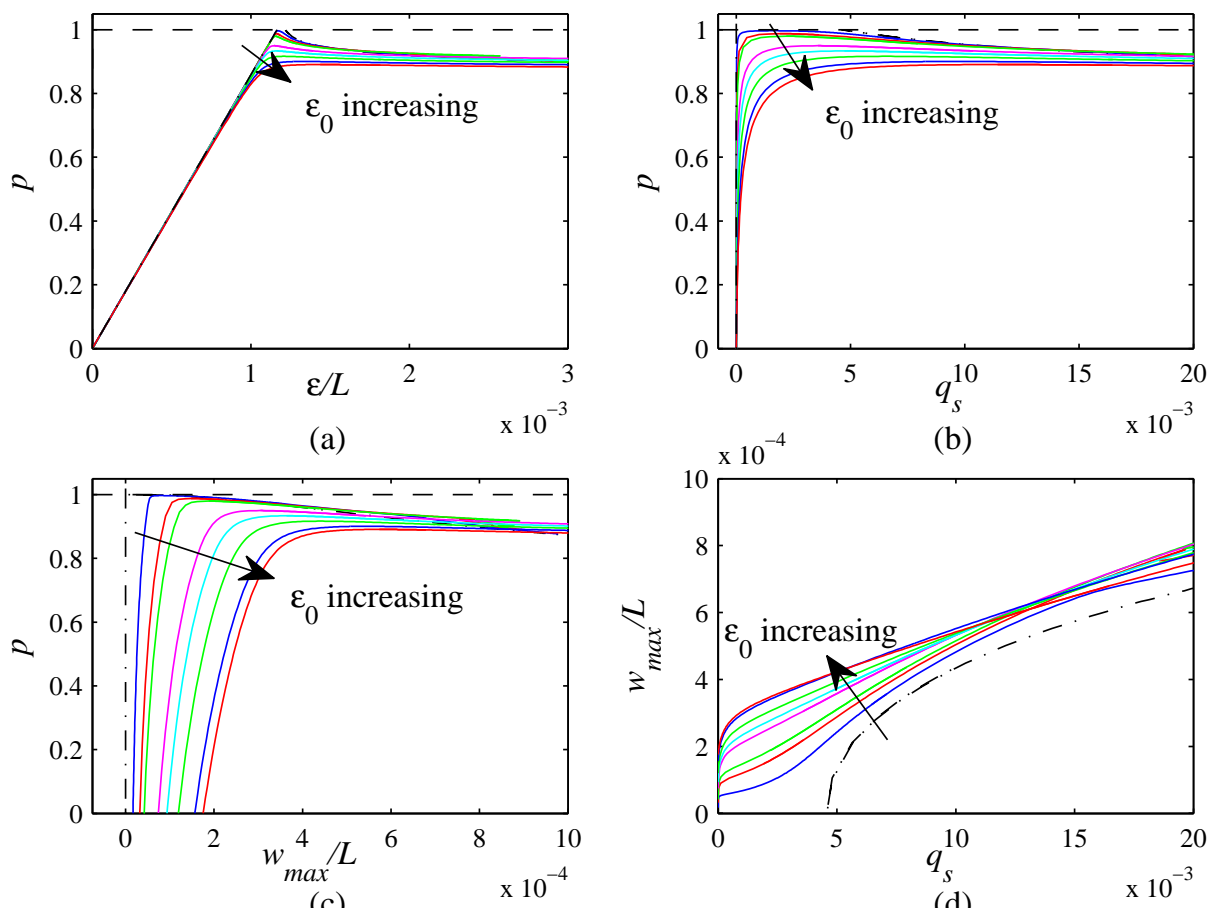

(c)

(d)

Figure 13: Equilibrium paths for the worst case localized imperfection cases. The family of curves are plotted for increasing values of normalized initial end-shortening $\mathcal{E}_{0} / L$ ranging from $1 \times 10^{-7}$ to $8 \times 10^{-5}$. Graphs show (a) the normalized load $p=P / P_{o}^{\mathrm{C}}$ versus the normalized total end-shortening $\mathcal{E} / L$, (b) $p$ versus the global mode amplitude $q_{s}$, (c) $p$ versus the maximum out-of-plane deflection $w_{\max } / L$ and (d) $w_{\max } / L$ versus $q_{s}$. The imperfect cases are shown in solid lines and the perfect case is shown with a dot-dashed line.

$A_{0}, \alpha$ and $\beta$ applied, corresponding to a selection of $\mathcal{E}_{0} / L$, ranging from $1 \times 10^{-7}$ to $8 \times 10^{-5}$. From the equilibrium paths, the decrease in the ultimate load for larger imperfections is apparent. It can also be seen that all equilibrium paths converge asymptotically to the 
same equilibrium state, thus it is expected that for large imperfections, the post-buckling response of the strut would be weakly stable if the struts were to remain elastic.

\subsubsection{Global and local imperfections: $q_{s 0}, q_{t 0}, w_{0} \neq 0$}

The I-section strut is now analysed for the case where both global and local initial imperfections are present. The global imperfection $q_{s 0}$ is kept at a constant value of $1 / 10000$ throughout, along with the corresponding $q_{t 0}$ value as calculated using Equation (43). The local imperfection parameters are then varied in a similar manner to the previous section in order to determine the worst case combination of $A_{0}, \alpha$ and $\beta$, i.e. the combination giving the lowest ultimate load. The ultimate loads that are found for normalized initial end-shortening values of $\mathcal{E}_{0} / L$ for the local imperfections ranging from $10^{-7}$ to $10^{-5}$ for both periodic and localized shapes and the equilibrium paths are plotted in Fig. 14, along
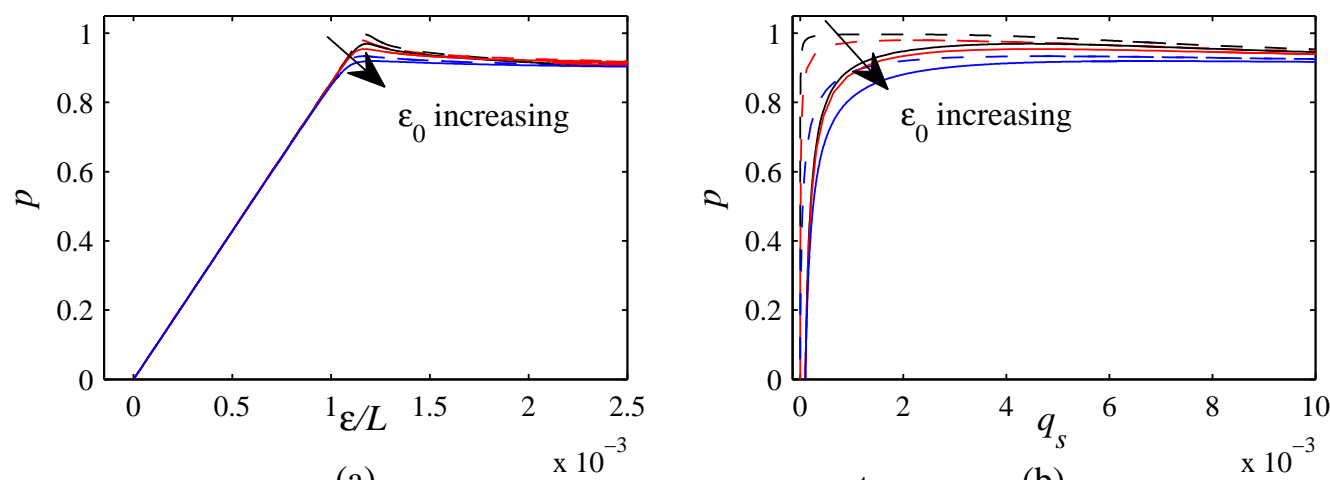

(a)

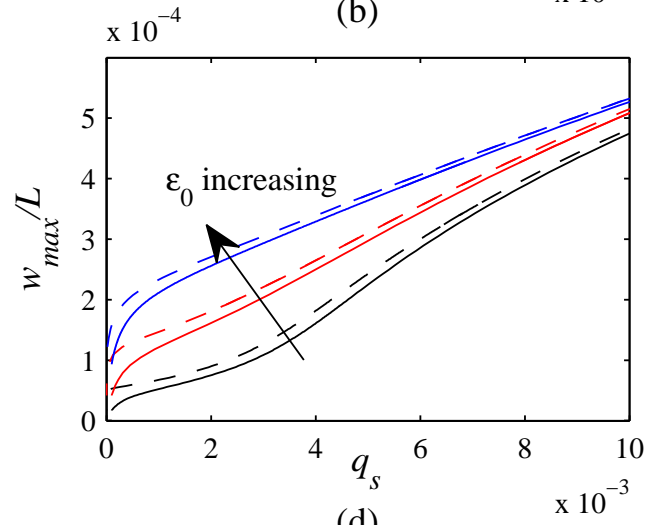

(c)

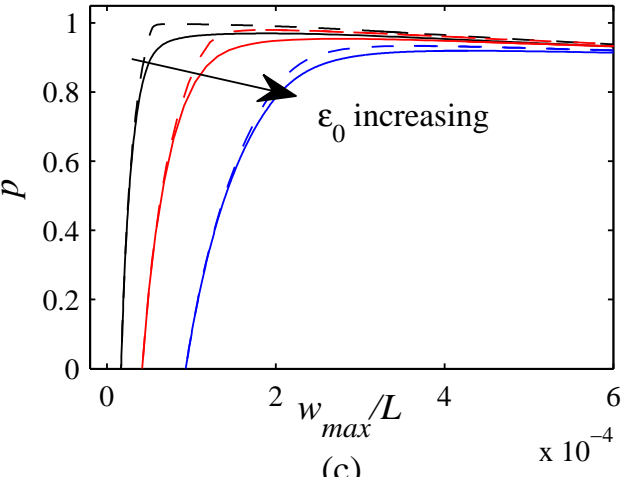

(d)

Figure 14: Equilibrium paths for the worst case localized imperfection with increasing initial end-shortening values $\mathcal{E}_{0}$, some of which include a constant global out-of-straightness of $q_{s 0}=1 / 10000$. Graphs show (a)(d) as described in Fig. 13. Cases with local imperfections only are shown with dashed lines and the corresponding paths that have the additional global imperfection $q_{s 0}$ are shown with solid lines.

with the paths obtained earlier for when only the local imperfection was present.

It is observed in Fig. 14 that the combination of the two types of imperfection has a greater effect in reducing the load capacity compared to the cases where global or local 
imperfections were present in isolation, as predicted. For smaller local imperfections, the same global imperfection has a greater effect in reducing the load capacity. For example, at an initial end-shortening of $\mathcal{E}_{0} / L=1 \times 10^{-7}$, a further $2.7 \%$ decrease in the ultimate load is observed with the addition of the global imperfection, whereas for a much larger local imperfection of $\mathcal{E}_{0} / L=1 \times 10^{-5}$, the same global imperfection only reduces the ultimate load further by $1.4 \%$. It can also be observed that this ultimate load occurs much later in the post-buckling path for the combined case when compared to the case where only local imperfections are present. Similar to the cases where only global or local imperfections are present, the combined cases result in equilibrium paths that converge asymptotically to the same equilibrium state, indicating that, again, reasonably large combined imperfections would also result in an approximately weakly stable post-buckling path for an elastic strut.

\subsubsection{FE model and validation}

Similar to the previous FE model devised within ABAQUS to analyse the local buckling load, a model is constructed using the same method to validate the nonlinear model with imperfections. An eigenvalue analysis is used initially to find the critical buckling modes of the strut and the corresponding load carrying capacities. The initial global and local buckling modes are then introduced as geometric imperfections into the structure and nonlinear Riks analysis is conducted. This is to compare the post-buckling response obtained from FE with the fully nonlinear analytical model presented currently.

To compare the FE model with the analytical model for the case where initial imperfections are present, care needs to be exercised. Results obtained from AUTO-07P solving the analytical model equations must implement, as far as is practicable, identical shapes of prescribed imperfections, for both the global out-of-straightness, as well as the local out-of-plane imperfection profile. The global out-of-straightness profile given by the linear eigenvalue analysis in ABAQUS is approximately a half-sine wave, which is quite satisfactorily modelled by the imperfection shape $q_{s 0}$. The amplitude of the global imperfection is set with $q_{s 0}=1 / 10000$ for the analytical model, but matching the local out-of-plane imperfection is slightly less straightforward.

The local imperfection profile of the flange found using linear eigenvalue analysis in ABAQUS can be extracted and plotted using MATLAB [37] and numerical integration can be used to determine the initial end-shortening $\mathcal{E}_{0}$ from the profile. This value can then be introduced into the analytical model. The $\beta$ and $\alpha$ values from the analytical imperfection shape can then be adjusted such that they match best with the FE imperfection profile, while producing the same initial end-shortening $\mathcal{E}_{0}$ value, thus ensuring the same size of imperfection is applied to both models - the best match is achieved when $\alpha=4.19$ and $\beta=29$. The $A_{0}$ value in the analytical model is set to $t_{f} / 10$ and the normalized initial endshortening for both models is $\mathcal{E}_{0} / L=3.46 \times 10^{-6}$. The initial local imperfection profiles for both the analytical and the FE models are shown in Fig. 15.

The results from the FE model are compared with the numerical solutions obtained from the analytical model, and equilibrium paths from both methods are presented in Fig. 16. The figure shows the analytical and FE models exhibiting good correlation, particularly in the neighbourhood of the limit point, where the two results match very well. The out- 


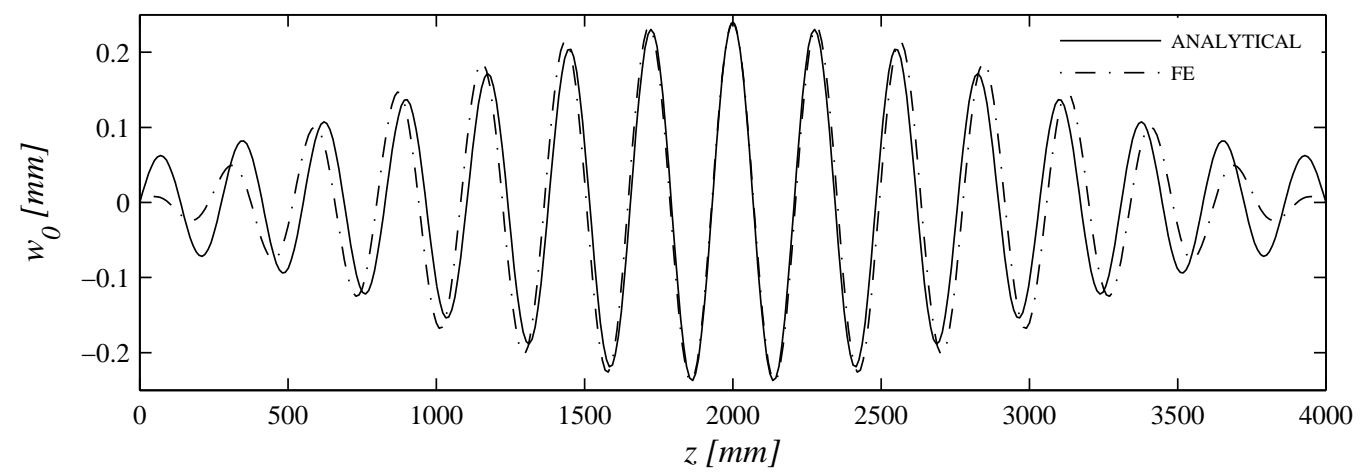

Figure 15: Local imperfection profile used in the validation study, shown for both the analytical model (solid line) and the FE model (dot-dashed line), such that the respective values of $\mathcal{E}_{0} / L$ match.

of-plane displacement profiles for both the flange and the web are also shown in Figs. 17 and 18. Note that for the analytical model, the out-of-plane displacement of the web is calculated by multiplying the profile of the flange by the factor $\kappa_{c}$, as defined earlier; again, excellent correlation is observed. At the respective ultimate loads, the out-of-plane displacement profiles for the analytical and FE models are almost identical in both the flange and the web. Recalling from Fig. 8 that, as the post-buckling path progresses, the analytical model for the perfect case exhibits a stiffer post-buckling response than the FE model, this is also found in the imperfect case. This is again primarily attributed to the assumption in the analytical model that the displacements of the less compressed flange are negligible, which generates a stiffer response, as seen previously for the perfect case. However, it is worth reiterating that the prediction of the ultimate load from the analytical model is still very good, implying that a practical prediction of the actual load carrying capacity can be established from the analytical model.

\section{Concluding remarks}

A series of nonlinear analytical models has been formulated from variational principles to investigate the interactive buckling of I-section struts where global buckling occurs about the strong axis of bending. These are subsequently validated within the commercial FE package ABAQUS. It was found that the assumption of a completely rigid flange-web connection in combination with approximate shape functions in the analytical model gave a significant over-prediction of the local buckling load. The analytical model was therefore calibrated by modifying the flange-web connection parameter $\kappa$ such that the local buckling loads matched those found from the FE model. This, in turn, improved the comparisons between the nonlinear analytical model subsequently presented and the FE model for the post-buckling response significantly.

Imperfection sensitivity of an I-section strut exhibiting an interaction between global buckling about the strong axis with local flange and web plate buckling was then studied. 


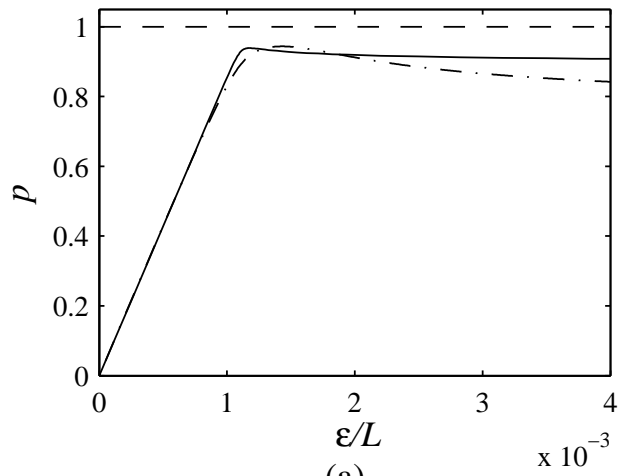

(a)

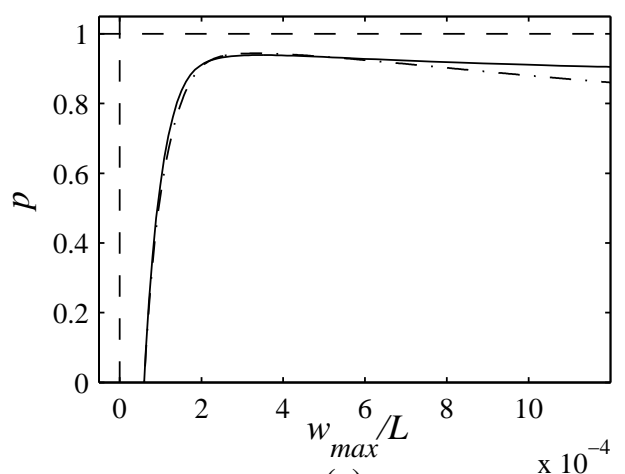

(c)
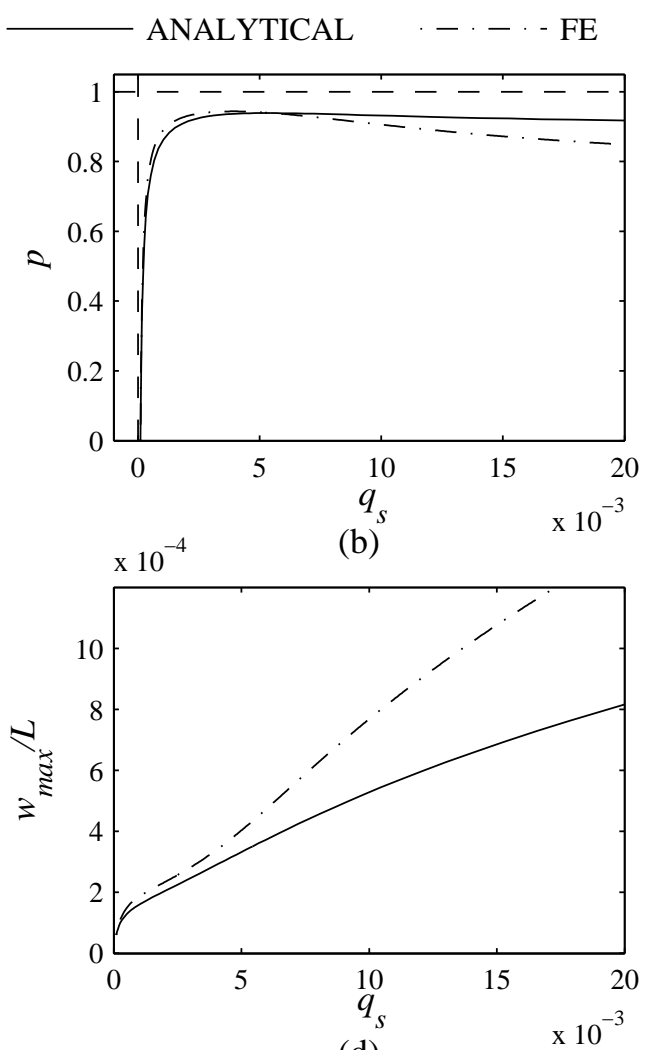

(d)

Figure 16: Numerical solutions of the equilibrium equations from the analytical model (solid line) and the FE solutions (dot-dashed line) for the imperfect strut with global and local initial imperfections, where $q_{s 0}=1 / 10000$ and $\mathcal{E}_{0} / L=3.46 \times 10^{-6}$. Graphs show (a)-(d) as described in Fig. 13.

The strut was found to be sensitive to all shapes of local imperfection, but lower ultimate loads were observed for the localized imperfection shapes. The model was validated using a nonlinear FE model, the results of which showed excellent comparisons for both equilibrium paths and mode shapes for the flange and web, particularly in the neighbourhood of the ultimate load where modal interactions are triggered. As the post-buckling path progressed, the analytical model exhibited a stiffer response, which is attributed mostly to the assumption that there is no out-of-plane displacement in the less compressed flange, even though the amplitude of these deflections is small is comparison with deflections elsewhere in the cross-section. In further studies, it would be desirable to incorporate the less compressed flange into the nonlinear analytical model such that more accurate results may be determined. This would, however, lead to a significantly more complex system of equations with the response of the two flanges coupling together, which did not appear in earlier related models $[16,32]$. In the current model, however, the ultimate load carrying capacity of the struts is predicted very well.

A study to investigate the parametric boundaries for where the strut makes the transi- 

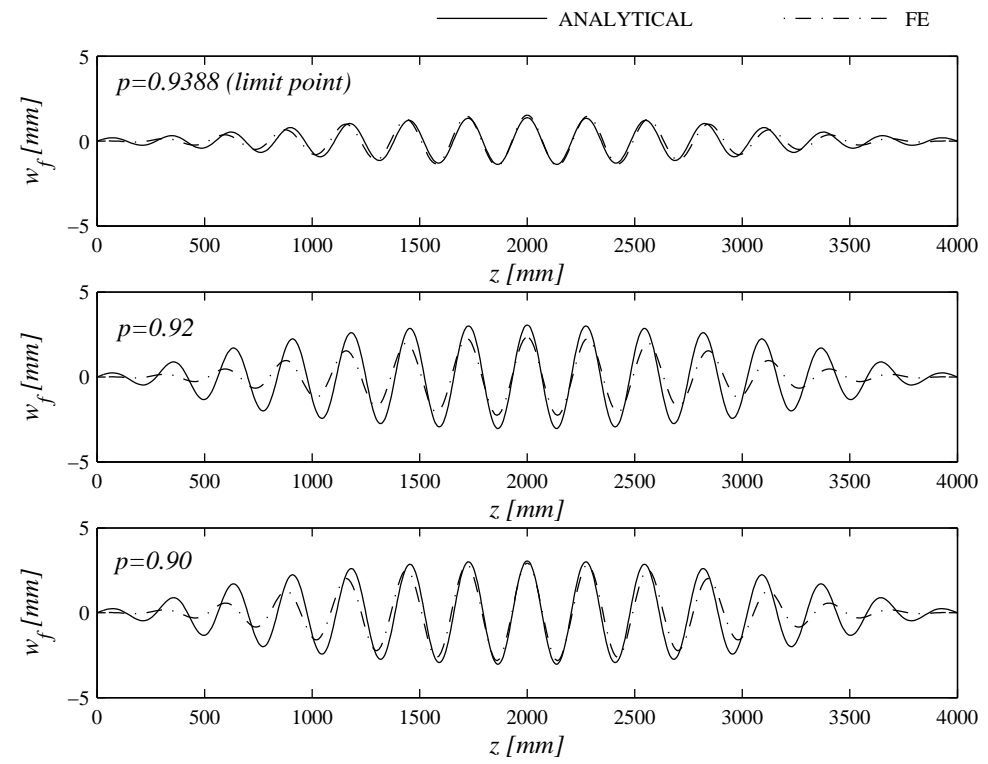

Figure 17: Comparisons of the out-of-plane displacement function $w_{f}$ of the flange between the numerical solution of the analytical model (solid line) and the FE model (dot-dashed line), for different loads in the post-buckling range for the imperfect strut.

tion from global buckling to local buckling being critical and the parametric ranges where modal interactions are significant would be desirable - this is currently under development and the authors hope to present this in the near future.

\section{Acknowledgement}

Financial support for the lead author was provided by the UK Engineering and Physical Sciences Research Council under the Doctoral Training Grant scheme.

\section{References}

[1] J. M. T. Thompson, G. W. Hunt, A general theory of elastic stability, John Wiley and Sons Ltd, London, UK, 1973.

[2] W. T. Koiter, M. Pignataro, An alternative approach to the interaction between local and overall buckling in stiffened panels, in: B. Budiansky (Ed.), Buckling of structures, International Union of Theoretical and Applied Mechanics, Springer Berlin Heidelberg, 1976, pp. 133-148.

[3] J. M. T. Thompson, G. W. Hunt, Elastic instability phenomena, John Wiley and Sons Ltd, Chichester, UK, 1984.

[4] V. Gioncu, General theory of coupled instabilities, Thin-Walled Structures 19 (2-4) (1994) 81-127. 

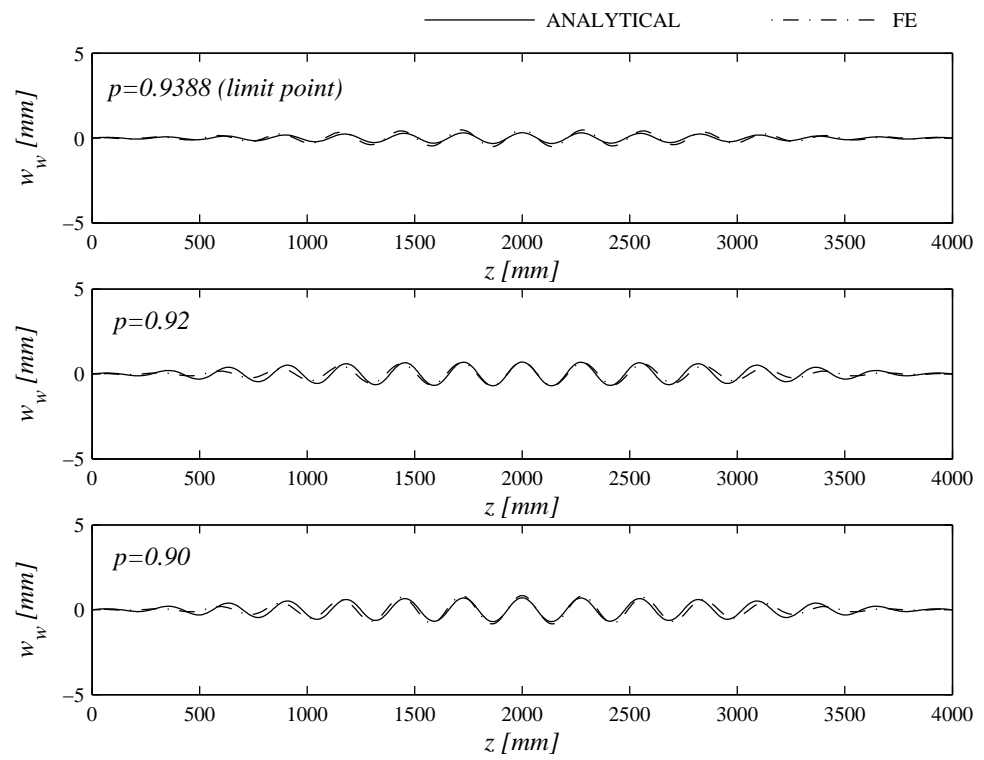

Figure 18: Comparisons of the out-of-plane displacement function $w_{w}$ of the web between the numerical solution of the analytical model (solid line) and the FE model (dot-dashed line), for different loads in the post-buckling range for the imperfect strut.

[5] G. W. Hunt, L. S. da Silva, G. M. E. Manzocchi, Interactive buckling in sandwich structures, Proceedings of the Royal Society A 417 (1988) 155-177.

[6] M.-A. Douville, P. Le Grognec, Exact analytical solutions for the local and global buckling of sandwich beam-columns under various loadings, International Journal of Solids and Structures 50 (16-17) (2013) 2597-2609.

[7] M. Pignataro, M. Pasca, P. Franchin, Post-buckling analysis of corrugated panels in the presence of multiple interacting modes, Thin-Walled Structures 36 (1) (2000) $47-66$.

[8] K. G. Ghavami, M. R. Khedmati, Numerical and experimental investigation on the compression behaviour of stiffened plates, Journal of Constructional Steel Research 62 (11) (2006) 1087-1100.

[9] N. Olhoff, A. P. Seyrenian, Bifurcation and post-buckling analysis of bimodal optimum columns, International Journal of Solids and Structures 45 (14-15) (2008) 3967-3995.

[10] G. J. Hancock, Interactive buckling in I-section columns, ASCE Journal of the Structural Division 107 (1) (1981) 165-179.

[11] B. W. Schafer, Local, distortional, and Euler buckling of thin-walled columns, ASCE Journal of Structural Engineering 128 (3) (2002) 289-299. 
[12] J. Becque, K. J. R. Rasmussen, Experimental investigation of the interaction of local and overall buckling of stainless steel I-columns, ASCE Journal of Structural Engineering 135 (11) (2009a) 1349-1356.

[13] J. Becque, K. J. R. Rasmussen, Numerical investigation of the interaction of local and overall buckling of stainless steel I-columns, ASCE Journal of Structural Engineering 135 (11) (2009b) 1340-1348.

[14] A. Van der Neut, The interaction of local buckling and column failure of thin-walled compression members, in: M. Hetenyi, W. G. Vincenti (Eds.), Proceedings of the 12th International Congress of Applied Mechanics, 1969, pp. 389-399.

[15] A. Van der Neut, The sensitivity of thin-walled compression members to column axis imperfections, International Journal of Solids and Structures 9 (1973) 999-1011.

[16] M. A. Wadee, M. Farsi, Local-global mode interaction in stringer-stiffened plates, Thin-Walled Structures 85 (2014) 419-430.

[17] M. A. Wadee, L. Bai, Cellular buckling in I-section struts, Thin-Walled Structures 81 (2014) 89-100.

[18] D. Dubina, V. Ungureanu, Instability mode interaction: From Van Der Neut model to ECBL approach, Thin-Walled Structures 81 (2014) 39-49.

[19] J. Loughlan, Thin-Walled Structures: Advances in Research, Design and Manufacturing Technology, Taylor and Francis, London, UK, 2004.

[20] E. L. Liu, M. A. Wadee, Interactively induced localization in thin-walled I-section struts buckling about the strong axis, Structures 4 (2015) 13-26.

[21] ABAQUS, Version 6.14, Dassault Systèmes, Providence RI, 2014.

[22] M. A. Wadee, Effects of periodic and localized imperfections on struts on nonlinear foundations and compression sandwich panels, International Journal of Solids and Structures 37 (8) (2000) 1191-1209.

[23] M. A. Wadee, M. Farsi, Imperfection sensitivity and geometric effects in stiffened plates susceptible to cellular buckling, Structures 3 (2015) 172-186.

[24] L. Bai, M. A. Wadee, Imperfection sensitivity of thin-walled I-section struts susceptible to cellular buckling, International Journal of Mechanical Sciences 104 (2015) 162-173.

[25] G. W. Hunt, M. A. Wadee, Localization and mode interaction in sandwich structures, Proceedings of the Royal Society A 454 (1972) (1998) 1197-1216.

[26] S. P. Timoshenko, J. M. Gere, Theory of elastic stability, McGraw-Hill, New York, USA, 1961. 
[27] M. K. Wadee, G. W. Hunt, A. I. M. Whiting, Asymptotic and Rayleigh-Ritz routes to localized buckling solutions in an elastic instability problem, Proceedings of the Royal Society A 453 (1965) (1997) 2085-2107.

[28] S. Timoshenko, S. Woinowsky-Krieger, Theory of plates and shells, McGraw-Hill, New York, USA, 1959.

[29] M. B. Monagan, K. O. Geddes, M. H. Heal, G. Labahn, S. M. Vorkoetter, J. McCarron, P. DeMarco, Maple 10 Programming Guide, Maplesoft, Waterloo ON, Canada, 2005.

[30] E. J. Doedel, B. E. Oldeman, AUTO-07P: Continuation and Bifurcation Software for Ordinary Differential Equations, Concordia University, Montreal, Canada, 2011.

[31] P. S. Bulson, The stability of flat plates, Chatto \& Windus, London, UK, 1970.

[32] L. Bai, M. A. Wadee, Mode interaction in thin-walled I-section struts with semi-rigid flange-web joints, International Journal of Non-Linear Mechanics 69 (2015) 71-83.

[33] E. Riks, An incremental approach to the solution of snapping and buckling problems, International Journal of Solids and Structures 15 (7) (1979) 529-551.

[34] C. J. Budd, G. W. Hunt, R. Kuske, Asymptotics of cellular buckling close to the Maxwell load, Proceedings of the Royal Society A 457 (2001) 2935-2964.

[35] M. A. Wadee, L. Gardner, Cellular buckling from mode interaction in I-beams under uniform bending, Proceedings of the Royal Society A 468 (2137) (2012) 245-268.

[36] M. A. Wadee, M. Farsi, Cellular buckling in stiffened plates, Proceedings of the Royal Society A 470 (2168) (2014) 20140094.

[37] MATLAB, version 7.10.0 (R2012a), The MathWorks Inc., Natick, Massachusetts, 2010. 